\title{
Density matrix of a quantum field in a particle-creating background
}

\author{
S.P. Gavrilov*, D.M. Gitman’ and J.L. Tomazelli ${ }^{\ddagger}$ \\ Instituto de Física, Universidade de São Paulo, \\ Caixa Postal 66318, CEP 05315-970 São Paulo, SP, Brazil
}

July 24, 2013

\begin{abstract}
We examine the time evolution of a quantized field in external backgrounds that violate the stability of vacuum (particle-creating backgrounds). Our purpose is to study the exact form of the final quantum state (the density operator at the final instant of time) that has emerged from a given arbitrary initial state (from a given arbitrary density operator at the initial time instant) in the course of evolution. We find a generating functional that allows one to obtain density operators for an arbitrary initial state. Averaging over states of the subsystem of antiparticles (particles), we obtain explicit forms of reduced density operators for the subsystem of particles (antiparticles). Analyzing one-particle correlation functions, we establish a one-to-one correspondence between these functions and the reduced density operators. It is shown that in the general case a presence of bosons (e.g., gluons) in the initial state increases the creation rate of the same type of bosons. We discuss the question (and its relation to the initial stage of quark-gluon plasma formation) whether a thermal form of one-particle distribution can appear even if the final state of the complete system is not in thermal equilibrium. In this respect, we discuss some cases when pair-creation by an electric-like field can mimic the one-particle thermal distribution. We apply our technics to some QFT problems in slowly varying electric-like backgrounds: electric, SU(3) chromoelectric, and metric. In particular, we analyze the time and temperature behavior of the mean numbers of created particles, provided that the effects of switching the external field on and off are negligible. It is demonstrated that at high temperatures and in slowly varying electric fields the rate of particle-creation is essentially time-dependent.
\end{abstract}

Keywords: external particle-creating field; evolution of an arbitrary initial state; creation rate; electric, SU(3) chromoelectric and metric fields; initial thermal distribution.

\section{Introduction}

The effect of particle creation from vacuum by an external background (vacuum instability in external fields) ranks among the most intriguing nonlinear phenomena in quantum theory. Its theoretical analysis must be nonperturbative, and its observation in experiment is to verify the applicability of a theory in the domain of superstrong fields. The study of this effect started in connection with the so-called Klein [1] paradox, and was carried on by Schwinger [2, who calculated the vacuum-to-vacuum transition probability in a constant electric field. A complete study of particle creation from vacuum by a constant electric field is presented in 3, 4. It should be noted that the effect can actually be observed as soon as the external field strength approaches the characteristic value (critical field) $E_{c}=m^{2} c^{3} /|e| \hbar \simeq 1,3$. $10^{16} \mathrm{~V} / \mathrm{cm}$. Although an actual possibility of creating these fields under laboratory conditions does not exist at present, $e^{+} e^{-}$-pair production by a slowly varying (external) electric field from vacuum is possibly relevant to phenomenology with the advent of a new laser technology, which may access the truly

\footnotetext{
* On leave from Department of general and experimental physics, Herzen State Pedagogical University of Russia, Moyka emb. 48, 191186 St. Petersburg, Russia; electronic address: gavrilovsergeyp@yahoo.com

${ }^{\dagger}$ Electronic address: gitman@dfn.if.usp.br

${ }_{\ddagger}^{\ddagger}$ Dept. Física, CCFM, Universidade Federal de Santa Catarina, CP 476, CEP 88010-970, Santa Catarina, SC, Brazil; electronic address: tomazelli@fsc.ufsc.br
} 
strong-field domain. Electron-positron pairs can also be created by perfectly perturbative processes in crossed laser beams, when there occurs the merging of several photons. The control over the dependence between the power and frequency of lasers makes in possible to determine which of these mechanisms is responsible for pair-creation. This topic is widely discussed [5] at SLAC and TESLA X-ray laser facilities. Such strong fields may be essential in astrophysics, where characteristic values of the electromagnetic and gravitational fields in the vicinity of black holes are enormous. Electric fields near a cosmic string can become extremely strong [6]. In this respect, one needs to mention that the Coulomb field of superheavy nuclei may create electron-positron pairs; see [7. Apart from purely QED problems, there arise problems of QFT in which vacuum instability in various external backgrounds plays an important role, for example, phase transitions in non-Abelian theories, the problem of boundary conditions, or the influence of topology on vacuum, the problem of a consistent vacuum construction in QCD and GUT, multiple particle-creation within the context of heavy-ion collisions, and so on. Particle creation by background metrics is important in black-hole physics [8, 9] and also in the study of the dynamics of the early Universe [10. Recently, it has also been recognized that the presence of a background electric field must be taken into account in string theory constructions; see, e.g., [11] and references therein.

Considerable attention has been recently focused on a non-perturbative parton production from vacuum by a classical chromoelectric field of SU(3) [12, 13] and $\mathrm{SU}(2)$ [14, in the framework of a modern version of the known chromoelectric flux tube model [15], the latter being an effective model for the confinement of quarks in QCD (previously pair-creation by a constant field has been calculated for SU(2) in [16] and for $\mathrm{SU}(3)$ in [17]). The model ensures a very good description of the phenomenology of hadron jets in high-energy $e^{+}-e^{-}$and $p-\bar{p}$ collision experiments (a further development of the basic model and phenomenological applications can be found in the review [18]). This model probably describes the initial stage of quark-gluon plasma formation reasonably well (in particular, the transversal spectrum of produced soft partons). Such a state may be produced at high-energy large-hadron colliders such as RHIC (Au-Au collisions at $\sqrt{s}=200 \mathrm{GeV})[19$ and $\mathrm{LHC}(\mathrm{Pb}-\mathrm{Pb}$ collisions at $\sqrt{s}=5,5 \mathrm{TeV})[20$. At present, this initial stage is related to an effective theory, the color glass condensate [21] (see also the review papers [22]), which is the coherent limit of quark-gluon plasma at high energies. In such a picture, after a nuclei collision, a strong classical chromo-electric-magnetic field is created due to relatively slow fluctuations of color density. Such a field is sufficiently uniform in the direction that is transversal to the beam direction and has a longitudinal chromoelectric component [14, 23. This component is much more intensive than the transversal component; see [14. Thus, the color glass condensate picture provides strong arguments in favour of the chromoelectric flux tube model and allows one to calculate field configurations in a tube. In particular, such a physical picture allows one to accept a quasi-constant chromoelectric field as a good approximation at the above-mentioned initial stage. It should be noted that experimental data on heavy-ion collisions that exist at present can be interpreted as quantum parton production by an external chromoelectric field from vacuum and many-particle states.

There is a considerable interest in particle-creation at finite temperatures and at a finite particle density, which is basically motivated by heavy-ion collisions, cosmological QCD phase transitions, and dark matter formation. For example, thermally-influenced pair-production in constant electric fields at the one-loop level has been searched for in $24,25,26,27$.

The above calculations have been carried out within the theory of a quantized field placed in an external background. A consistent description of a complete QED (interacting quantum electromagnetic and matter fields in a particle-creating background) with unstable vacuum that treats the interaction with external backgrounds nonperturbatively has been developed in [28. Possible generalizations of the formalism to external gravitational and non-Abelian gauge fields have been presented in 29, 30]; see also [31]. An attempt to extend this technics to the thermal case has been taken in [32]. Calculating particle creation by black-hole metric, Hawking has discovered that the density matrix of created particles at spatial infinity has a thermal character. The question arises, is such a character related to the particlecreation mechanism in general, or to the gravitational origin of the background? A way to answer this question is to elaborate an adequate technique which could allow one to include arbitrary mixed initial states, in particular, thermal initial states, in the corresponding particle creation formalism [28.

In this article, we present a development of the particle-creation formalism [28, that is capable to answer some of the above questions. We examine the time evolution of a quantized field (bosonic or fermionic) in external backgrounds that violates the stability of vacuum. In fact, we deal with a quadratic field theory of noninteracting (between themselves) particles. Our purpose is to study the exact form of the final quantum state (the density operator at the final instant of time) that has emerged from a given arbitrary initial state (from a given arbitrary density operator at the initial time instant) in the course 
of evolution.

The article is organized as follows. Section 2 has an original, albeit rater technical character. There, we derive exact expressions for density operators (more appropriate for the generating density operator) by applying the path integration method. Some necessary formulas are placed in Appendix. In Section 3 , having an exact expression for the generating density operator, we obtain reduced density operators for the subsystems of particles and antiparticles. We introduce and calculate the one-particle correlation functions and establish a one-to-one correspondence between these functions and the reduced density operators. In particular, this allows us to restore the reduced density operator of the complete system starting from the one-particle distributions (of course, this is possible only in the model under consideration being a quadratic theory). It is demonstrated that in the general case the presence of bosons (e.g., gluons) in the initial state increases the creation of the same kind of bosons. We discuss in detail the question (and its relation to the initial stage of quark-gluon plasma formation) whether the thermal form of the one-particle distribution can appear even if the final state of the complete system is not in thermal equilibrium. In Section 4, we discuss the expressions we obtain for the density operators and one-particle distributions in electric-like backgrounds: electric, SU(3) chromoelectric, and metric. In particular, we analyze the density operators and one-particle distributions in the so-called $T$-constant electric background (a field exists during a finite period of time $T$ ), and demonstrate how such a problem is related to particle creation by gravitational fields (Hawking's effect). We present some examples when pair-creation by an electric-like field can mimic the one-particle thermal distribution. Then, we analyze the time and temperature behavior of particle-creation when the effects of switching on and off are negligible. In particular, we demonstrate that at high temperatures the production rate is non-trivially time-dependent. This result has to be taken into account at high temperatures.

\section{Density operator in pair-creating backgrounds}

We consider a quantum field $\psi(x)$ in an external background. The quantum field can be scalar, spinor, etc., and the background can be the classical electromagnetic, Yang-Mills, or gravitational field. In the general case, the background is intense, time-dependent, and violates the stability of vacuum. Such a background must be treated nonperturbatively. We plan to follow the formalism proposed in [28].

\subsection{Some relevant relations}

It is assumed that there exists a set of creation and annihilation operators $a_{n}^{\dagger}\left(t_{i n}\right), a_{n}\left(t_{i n}\right)$ of particles $a_{n}^{\dagger}\left(t_{i n}\right), a_{n}\left(t_{i n}\right)$, and antiparticles $b_{n}^{\dagger}\left(t_{i n}\right), b_{n}\left(t_{i n}\right)$, respectively, at the initial time instant $t_{\text {in }}\left(t_{\text {in }} \rightarrow-\infty\right)$, and a set of creation and annihilation operators of particles, $a_{n}^{\dagger}\left(t_{\text {out }}\right), a_{n}\left(t_{\text {out }}\right)$, and antiparticles, $b_{n}^{\dagger}\left(t_{\text {out }}\right)$, $b_{n}\left(t_{\text {out }}\right)$, at the final time instant $t_{\text {out }}\left(t_{\text {out }} \rightarrow \infty\right)$. By $n$ we denote a complete set of possible quantum numbers. The total Hamiltonian $\hat{H}(t)$ of the quantized field under consideration is diagonalized (and has a canonical form) in terms of the first set at the initial time instant, and is diagonalized (and has a canonical form) in terms of the second set at the final time instant. Nonzero (anti)commutator 1 are given by

$$
\begin{aligned}
& {\left[a_{n}\left(t_{\text {in }}\right), a_{m}^{\dagger}\left(t_{\text {in }}\right)\right]_{ \pm}=\left[a_{n}\left(t_{\text {out }}\right), a_{m}^{\dagger}\left(t_{\text {out }}\right)\right]_{ \pm}} \\
& =\left[b_{n}\left(t_{\text {in }}\right), b_{m}^{\dagger}\left(t_{\text {in }}\right)\right]_{ \pm}=\left[b_{n}\left(t_{\text {out }}\right), b_{m}^{\dagger}\left(t_{\text {out }}\right)\right]_{ \pm}=\delta_{n m} .
\end{aligned}
$$

The vacuum states $\left|0, t_{\text {in }}\right\rangle$ at $t_{\text {in }}$ and $\left|0, t_{\text {out }}\right\rangle$ at $t_{\text {out }}$ are defined as usual:

$$
a\left(t_{\text {in }}\right)\left|0, t_{\text {in }}\right\rangle=b\left(t_{\text {in }}\right)\left|0, t_{\text {in }}\right\rangle=0, a\left(t_{\text {out }}\right)\left|0, t_{\text {out }}\right\rangle=b\left(t_{\text {out }}\right)\left|0, t_{\text {out }}\right\rangle=0 .
$$

Therefore, we define these states as the states that minimize the mean value of the Hamiltonian $\hat{H}(t)$ at $t=t_{\text {in }}$ and $t=t_{\text {out }}$, respectively. According to the general princilples of quantum theory, the probability amplitude of transiltion from a certain initial state $b_{m}^{\dagger}\left(t_{i n}\right) \ldots a_{n}^{\dagger}\left(t_{i n}\right)\left|0, t_{i n}\right\rangle$ to a certain final state $b_{m^{\prime}}^{\dagger}\left(t_{\text {out }}\right) \ldots a_{n^{\prime}}^{\dagger}\left(t_{\text {out }}\right)\left|0, t_{\text {out }}\right\rangle$ in the Schrödinger picture has the form

$$
\left\langle 0, t_{\text {out }}\left|a_{n^{\prime}}\left(t_{\text {out }}\right) \ldots b_{m^{\prime}}\left(t_{\text {out }}\right) U\left(t_{\text {out }}, t_{\text {in }}\right) b_{m}^{\dagger}\left(t_{\text {in }}\right) \ldots a_{n}^{\dagger}\left(t_{\text {in }}\right)\right| 0, t_{\text {in }}\right\rangle,
$$

\footnotetext{
${ }^{1}$ The subscript - denotes the commutator (in the Bose case), whereas the subscript + denotes the anticommutator (in the Fermi case)
} 
where $U\left(t, t^{\prime}\right)$ is a unitary evolution operator of the system. Let $\hat{\rho}\left(t_{i n}\right)=\rho\left(a^{\dagger}\left(t_{i n}\right), a\left(t_{i n}\right), b^{\dagger}\left(t_{i n}\right), b\left(t_{i n}\right)\right)$, $\operatorname{tr} \hat{\rho}\left(t_{i n}\right)=1$, be the density operator of the system under consideration at the initial time instant. As the system develops in time, this density operator becomes $\hat{\rho}\left(t_{\text {out }}\right)$ for antiparticles at the final instant of time:

$$
\hat{\rho}\left(t_{\text {out }}\right)=U\left(t_{\text {out }}, t_{\text {in }}\right) \hat{\rho}\left(t_{\text {in }}\right) U^{\dagger}\left(t_{\text {out }}, t_{\text {in }}\right) .
$$

To pass to the Heisenberg picture, we introduce finite-time evolution operators $\Omega_{( \pm)}$,

$$
\Omega_{(+)}=U\left(0, t_{\text {in }}\right), \Omega_{(-)}=U\left(0, t_{\text {out }}\right), U\left(t_{\text {out }}, t_{\text {in }}\right)=\Omega_{(-)}^{\dagger} \Omega_{(+)} .
$$

We then define a set of creation and annihilation operators $a_{n}^{\dagger}(i n), a_{n}(i n)$ of $i n$-particles, as well as similar operators $b_{n}^{\dagger}(i n), b_{n}(i n)$ of $i n$-antiparticles, a corresponding $i n$-vacuum $|0, i n\rangle$, a set of creation and annihilation operators $a_{n}^{\dagger}, a_{n}$, of out-particles and similar operators $b_{n}^{\dagger}, b_{n}$ of out-antiparticles, and a corresponding out-vacuum $|0\rangle$,

$$
\begin{aligned}
& \left(a^{\dagger}(\text { in }), a(\text { in }), b^{\dagger}(\text { in }), b(\text { in })\right)=\Omega_{(+)}\left(a^{\dagger}\left(t_{\text {in }}\right), a\left(t_{\text {in }}\right), b^{\dagger}\left(t_{\text {in }}\right), b\left(t_{\text {in }}\right)\right) \Omega_{(+)}^{\dagger}, \\
& \left(a^{\dagger}, a, b^{\dagger}, b\right)=\Omega_{(-)}\left(a^{\dagger}\left(t_{\text {out }}\right), a\left(t_{\text {out }}\right), b^{\dagger}\left(t_{\text {out }}\right), b\left(t_{\text {out }}\right)\right) \Omega_{(-)}^{\dagger}, \\
& \mid 0, \text { in }\rangle=\Omega_{(+)}\left|0, t_{\text {in }}\right\rangle,|0\rangle=\Omega_{(-)}\left|0, t_{\text {out }}\right\rangle .
\end{aligned}
$$

The in- and out-operators obey the canonical commutation relations (11).

The entire information concerning the processes of particle creation, annihilation and scattering is contained in the elementary probability amplitudes

$$
\begin{aligned}
& w(+\mid+)_{m n}=c_{v}^{-1}\left\langle 0\left|a_{m} a_{n}^{\dagger}(i n)\right| 0, i n\right\rangle, \\
& w(-\mid-)_{n m}=c_{v}^{-1}\left\langle 0\left|b_{m} b_{n}^{\dagger}(i n)\right| 0, i n\right\rangle, \\
& w(0 \mid-+)_{n m}=c_{v}^{-1}\left\langle 0\left|b_{n}^{\dagger}(i n) a_{m}^{\dagger}(i n)\right| 0, i n\right\rangle, \\
& w(+-\mid 0)_{m n}=c_{v}^{-1}\left\langle 0\left|a_{m} b_{n}\right| 0, i n\right\rangle,
\end{aligned}
$$

where $c_{v}$ is the vacuum-to-vacuum transition amplitude, $c_{v}=\langle 0 \mid 0, i n\rangle$.

The sets of in and out-operators are related to each other by a linear canonical transformation (it is sometimes called the Bogolyubov transformation). As has been demonstrated in the general case, such a relation has the form (see [28])

$$
\begin{aligned}
& V\left(a^{\dagger}, a, b^{\dagger}, b\right) V^{\dagger}=\left(a^{\dagger}(i n), a(i n), b^{\dagger}(i n), b(i n)\right) \\
& |0, i n\rangle=V|0\rangle \quad\left(c_{v}=\langle 0|V| 0\rangle\right)
\end{aligned}
$$

where a unitary operator $V$ has the form

$$
V=v_{4} v_{3} v_{2} v_{1}
$$

and 2

$$
\begin{aligned}
& v_{1}=\exp \{-\kappa b w(0 \mid-+) a\}, v_{2}=\exp \left\{a^{\dagger} \ln w(+\mid+) a\right\}, \\
& v_{3}=\exp \left\{-\kappa b \ln w(-\mid-) b^{\dagger}\right\}, v_{4}=\exp \left\{-\kappa a^{\dagger} w(+-\mid 0) b^{\dagger}\right\}, \\
& \kappa=\left\{\begin{array}{l}
1 \text { Fermi case } \\
-1 \text { Bose case }
\end{array} .\right.
\end{aligned}
$$

Using this expression for $V$, one can find

$$
c_{v}=\langle 0|V| 0\rangle=\exp \{-\kappa \operatorname{tr} \ln w(-\mid-)\} .
$$

The density operator $\check{\rho}$ of the system under consideration in the Heisenberg picture is defined as

$$
\begin{aligned}
& \check{\rho}=\Omega_{(+)} \hat{\rho}\left(t_{\text {in }}\right) \Omega_{(+)}^{\dagger}=\rho\left(a^{\dagger}(\text { in }), a(\text { in }), b^{\dagger}(\text { in }), b(\text { in })\right), \\
& \check{\rho}=\Omega_{(-)} \hat{\rho}\left(t_{\text {out }}\right) \Omega_{(-)}^{\dagger}, \operatorname{tr} \check{\rho}=1 .
\end{aligned}
$$

\footnotetext{
${ }^{2}$ We use condenced notation, for example,

$$
b w(0 \mid-+) a=\sum_{n, m} b_{n} w(0 \mid-+)_{n m} a_{m} .
$$
}


Suppose a physical quantity is given by an operator $\hat{F}\left(t_{\text {out }}\right)$ at the final time instant as

$$
\hat{F}\left(t_{\text {out }}\right)=F\left(a^{\dagger}\left(t_{\text {out }}\right), a\left(t_{\text {out }}\right), b^{\dagger}\left(t_{\text {out }}\right), b\left(t_{\text {out }}\right)\right) .
$$

Then its mean value at the final instant of time is given by

$$
\langle F\rangle=\operatorname{tr}\left[\hat{F}\left(t_{\text {out }}\right) \hat{\rho}\left(t_{\text {out }}\right)\right]=\operatorname{tr}[\check{F} \check{\rho}],
$$

where

$$
\check{F}=\Omega_{(-)} \hat{F}\left(t_{\text {out }}\right) \Omega_{(-)}^{\dagger}=F\left(a^{\dagger}, a, b^{\dagger}, b\right)
$$

is the operator of a physical quantity in the Heisenberg representation 3 .

The amplitudes (44) can be calculated with the help of some appropriate sets of solutions of the corresponding relativistic wave equation (RWE) with an external field (Klein-Gordon, Dirac, linearized Yang-Mills), see [28, 30, as follows. Namely, the $i n$-particles are associated with a complete set (inset) of solutions of the RWE $\left\{{ }_{\zeta} \psi_{n}(x)\right\}$ with asymptotics ${ }_{\zeta} \psi_{n}\left(t_{i n}, \mathbf{x}\right)$ at the initial time-instant $t_{i n}$ being eigenvectors of the corresponding one-particle Hamiltonian $\mathcal{H}(t)$,

$$
\mathcal{H}\left(t_{i n}\right)_{\zeta} \psi_{n}\left(t_{i n}, \mathbf{x}\right)=\zeta \varepsilon_{n}^{(\zeta)}{ }_{\zeta} \psi_{n}\left(t_{i n}, \mathbf{x}\right),
$$

where $\varepsilon_{n}^{(\zeta)}$ are the energies of $i n$-particles in a state specified by a complete set of quantum numbers $n$, and $\varepsilon_{n}^{( \pm)}>0$. Here and elsewhere, $\zeta= \pm$, being $(+)$ for particles and $(-)$ for antiparticles. For the sake of simplicity of exposition, we use such a gauge-fixing of the external field that condition (13) should have the same form as it has in the absence of the external field. The out-particles are associated with the complete out-set of solutions $\left\{{ }^{\zeta} \psi_{n}(x)\right\}$ of the RWE with asymptotics ${ }^{\zeta} \psi_{n}\left(t_{\text {out }}, \mathbf{x}\right)$ at $t_{\text {out }}$ being eigenvectors of the Hamiltonian $\mathcal{H}(t)$ at $t_{\text {out }}$, namely,

$$
\mathcal{H}\left(t_{\text {out }}\right){ }^{\zeta} \psi_{n}\left(t_{\text {out }}, \mathbf{x}\right)=\zeta \tilde{\varepsilon}_{n}^{(\zeta) \zeta} \psi_{n}\left(t_{\text {out }}, \mathbf{x}\right),
$$

where $\tilde{\varepsilon}_{n}^{( \pm)}$are the energies of out-particles in a state specified by a complete set of quantum numbers $n$, and $\tilde{\varepsilon}_{n}^{( \pm)}>0$. We suppose that the external field is such that soluitons of this eigevalue problem actually exist.

The out-set can be decomposed in the $i n$-set as follows:

$$
{ }^{\zeta} \psi(x)={ }_{+} \psi(x) G\left(+\left.\right|^{\zeta}\right)+\kappa_{-} \psi(x) G\left(-\left.\right|^{\zeta}\right),
$$

where $\kappa=+1$ for fermions and $\kappa=-1$ for bosons. The decomposition coefficients $G\left(\zeta_{\zeta} \zeta^{\prime}\right)$ are expressed via inner products of these sets, $G\left(\zeta_{\zeta} \zeta^{\prime}\right)_{m n}=\left({ }_{\zeta} \psi_{m}, \zeta^{\prime} \psi_{n}\right)$. These coefficients obey the unitarity relations

$$
\begin{aligned}
& G\left(\left.{ }^{\zeta}\right|_{+}\right) G\left(\left.{ }_{+}\right|^{\zeta}\right)+\kappa G\left(\left.{ }^{\zeta}\right|_{-}\right) G\left(\left.{ }_{-}\right|^{\zeta}\right)=\zeta^{\frac{1-\kappa}{2}}, \\
& G\left(\left.\zeta\right|^{+}\right) G\left(\left.{ }^{+}\right|_{\zeta}\right)+\kappa G\left(\left.{ }_{\zeta}\right|^{-}\right) G\left(\left.{ }^{-}\right|_{\zeta}\right)=\zeta^{\frac{1-\kappa}{2}}, \\
& G\left(\left.{ }_{+}\right|^{+}\right) G\left(\left.{ }^{+}\right|_{-}\right)+\kappa G\left(\left.{ }_{+}\right|^{-}\right) G\left(\left.{ }^{-}\right|_{-}\right)=0, \\
& G\left(\left.{ }^{+}\right|_{+}\right) G\left(\left.{ }_{+}\right|^{-}\right)+\kappa G\left(\left.{ }^{+}\right|_{-}\right) G\left(\left.{ }_{-}\right|^{-}\right)=0,
\end{aligned}
$$

that follow from the normalization conditions for the solutions. Here, the notation $G\left(\left.\zeta^{\prime}\right|_{\zeta}\right)=G\left(\left.{ }_{\zeta}\right|^{\zeta^{\prime}}\right)^{\dagger}$ has been used.

The quantum Heisenberg field $\check{\psi}(x)$ can be expressed both from the creation and annihilation operators of in-particles and from the creation and annihilation operators of out-particles:

$$
\check{\psi}(x)={ }_{+} \psi(x) a(\text { in })+{ }_{-} \psi(x) b^{\dagger}(\text { in })={ }^{+} \psi(x) a+{ }^{-} \psi(x) b^{\dagger} .
$$

This makes it possible to express the linear canonical transformation between the sets of in and outoperators in terms of the coefficients $G\left(\left.\zeta^{\prime}\right|_{\zeta}\right)$,

$$
\begin{aligned}
a & =G\left(\left.{ }^{+}\right|_{+}\right) a(i n)+G\left(\left.{ }^{+}\right|_{-}\right) b^{\dagger}(i n), \\
\kappa b^{\dagger} & =G\left(\left.{ }^{-}\right|_{+}\right) a(i n)+G\left(\left.{ }^{-}\right|_{-}\right) b^{\dagger}(i n),
\end{aligned}
$$

\footnotetext{
${ }^{3}$ All operators in the Heisenberg representation are denoted by the turned over hat in what follows, e.g. $\check{A}$.
} 
and their Hermitian conjugated ones. Using relations (17), one finds the amplitudes (4) in the form:

$$
\begin{aligned}
& w(+\mid+)=G\left(\left.{ }_{+}\right|^{+}\right)^{-1}, w(-\mid-)=\kappa G\left(\left.{ }^{-}\right|_{-}\right)^{-1}, \\
& w(0 \mid-+)=-G\left(\left.{ }^{-}\right|_{-}\right)^{-1} G\left(\left(\left.^{-}\right|_{+}\right)=\kappa G\left(\left.{ }_{-}\right|^{+}\right) G\left(\left.{ }_{+}\right|^{+}\right)^{-1},\right. \\
& w(+-\mid 0)=\kappa G\left(\left.{ }_{+}\right|^{+}\right)^{-1} G\left(\left.{ }_{+}\right|^{-}\right)=-G\left(\left.{ }^{+}\right|_{-}\right) G\left(\left.{ }^{-}\right|_{-}\right)^{-1} .
\end{aligned}
$$

Using the same relations, one finds that the differential mean numbers $\aleph_{m}^{(\zeta)}$ of particles/antiparticle created from vacuum are

$$
\begin{aligned}
& \aleph_{m}^{(+)}=\left\langle 0, i n\left|a_{m}^{\dagger} a_{m}\right| 0, i n\right\rangle=\left[G\left(\left.{ }^{+}\right|_{-}\right) G\left(\left.{ }_{-}\right|^{+}\right)\right]_{m m}, \\
& \aleph_{m}^{(-)}=\left\langle 0, i n\left|b_{m}^{\dagger} b_{m}\right| 0, i n\right\rangle=\left[G\left(\left.{ }^{-}\right|_{+}\right) G\left(\left.{ }_{+}\right|^{-}\right)\right]_{m m} .
\end{aligned}
$$

Using the unitarity relations (16) and representation (8), we find that the probability $P^{v}$ of a vacuum to remain a vacuum is given by

$$
P^{v}=\left|c_{v}\right|^{2}=\exp \left\{\kappa \operatorname{tr} \ln \left[1-\kappa G\left(\left.{ }^{-}\right|_{+}\right) G\left(+\left.\right|^{-}\right)\right]\right\} .
$$

Therefore, we can see that the non-stability of vacuum in external fields is mainfest in the fact that $P^{v}<1$, which is in one-to-one-correspondence with the fact that the differential mean numbers $\aleph_{m}^{(\zeta)}$ are different from zero. We can now establish the following general property. Let the time-dependent external field turn on at the time instant $t_{1}$ and turn off at the time instant $t_{2}$, and in general behaves arbitrarily, on condition that the solutions of problems (13) and (14) do exist. Then, the $i n$-set of solutions $\left\{{ }_{\zeta} \psi_{n}(x)\right\}$ is defined equally for any solution of the time instant $t_{i n}<t_{1}$, and the out-set of solutions $\left\{{ }^{\zeta} \psi_{n}(x)\right\}$ is defined equally for any choice of the time instant $t_{\text {out }}>t_{2}$. Therefore, the coefficients $G\left(\left.\zeta^{\prime}\right|_{\zeta}\right)$, and consequently all the above-calculated amplitudes, depend only on the dependence of the external field at the finite interval of time between $t_{1}$ and $t_{2}$. This also implies that the $i n$ - and out- vacua, as well as the $i n$ - and out-operators of creation and annihilation, do not depend on the choice of the time instant $t_{i n}$ and $t_{\text {out }}$, respectively, if $t_{\text {in }}<t_{1}$ and $t_{\text {out }}>t_{2}$.

\subsection{Generating density operator}

We introduce the following generating operator $\check{R}(J)$ :

$$
\begin{aligned}
& \check{R}(J)=\frac{1}{Z} \underline{\check{R}}(J), \operatorname{tr} \check{R}(J)=1, \\
& \check{\underline{R}}(J)=\mathcal{N}_{i n} \exp \left[a^{\dagger}(i n)\left(\mathbb{J}^{(+)}-1\right) a(i n)+b^{\dagger}(i n)\left(\mathbb{J}^{(-)}-1\right) b(i n)\right],
\end{aligned}
$$

where the Grassmann-even variables $J=\left(J_{n}^{(\zeta)}\right)$ are sources; $\mathbb{J}_{m n}^{(\zeta)}=\delta_{m n} J_{n}^{(\zeta)} ; \mathcal{N}_{i n}$ is the sign of the normal form with respect to the $i n$-vacuum, and $Z=\operatorname{tr} \underline{\check{R}}(J)$ is a normalization factor (statistical sum).

In order to complete the calculation, it is efficient to use the path integral representation. In the fermion case, we use a path integral over anticommuting (Grassmann) variables, which is understood as Berezin's integral [33] at $\kappa=1$,

$$
: e^{-\kappa a^{\dagger} K a}:=\operatorname{det} K^{\kappa}: \int \exp \left\{\kappa \lambda^{*} K^{-1} \lambda+a^{\dagger} \lambda+\lambda^{*} a\right\} \Pi d \lambda^{*} d \lambda:
$$

where $a^{\dagger}, a$ are some creation and annihilation operators, and : ...: realizes the normal form of the operators $a^{\dagger}, a$. All operators $a^{\dagger}$ and $a$ can be considered as Grassmann-odd variables under the normal form; therefore, we can calculate the complete path integral (22) as a Gaussian integral over Grassmannodd variables. In the boson case, we use the path integral (22) over commuting variables at $\kappa=-1$. In this case, we can regard all the operators $a^{\dagger}$ and $a$ as bosonic (ordinary) variables under the normal form sign, so that the path integral in (22) is a usual Gaussian path integral, where $\lambda^{*} K^{-1} \lambda>0$.

Note that the trace of the normal product of creation and annihilation operators can be calculated by using the path integral representation, according to (110), see Appendix. For example, by calculating $Z$ we obtain

$$
Z=\exp \left\{\kappa \sum_{n}\left[\ln \left(1+\kappa \mathbb{J}^{(+)}\right)\right]_{n n}+\kappa \sum_{m}\left[\ln \left(1+\kappa \mathbb{J}^{(-)}\right)\right]_{m m}\right\} .
$$


Having at our disposal the generating operator (21), we can obtain the different density operators (in the Heisenberg representation) corresponding to different initial states of the system. We represent some examples below:

a) By setting $J=0$, we obtain a density operator $\check{\rho}_{v}$ of the system that is found in the pure vacuum state at the initial time instant,

$$
\check{\rho}_{v}=\check{R}(0) .
$$

Indeed, using relation (108) from Appendix, we obtain

$$
\check{\rho}_{v}=\mathcal{N}_{i n} \exp \left\{-\left[a^{\dagger}(i n) a(i n)+b^{\dagger}(i n) b(i n)\right]\right\}=|0, i n\rangle\langle 0, i n| .
$$

In addition, we define the following generating functional of moments:

$$
\begin{aligned}
& \Phi^{v}(J)=\left\langle 0, i n\left|\exp \left[a^{\dagger} \mathbb{J}^{(+)} a+b^{\dagger} \mathbb{J}^{(-)} b\right]\right| 0, i n\right\rangle=\operatorname{tr} \check{\phi}(J), \\
& \check{\phi}(J)=\exp \left[a^{\dagger} \mathbb{J}^{(+)} a+b^{\dagger} \mathbb{J}^{(-)} b\right] \check{\rho}_{v},
\end{aligned}
$$

which is useful to investigate the final state evolved from vacuum at the initial time instant.

b) The density operator $\check{\rho}_{\{m\}_{M} ;\{n\}_{N}}$ of the system which is found in a pure state with $M$ particles and $N$ antiparticles (with the quantum numbers $\left\{m_{1}, \ldots, m_{M}\right\}=\{m\}_{M}$ and $\left\{n_{1}, \ldots, n_{N}\right\}=\{n\}_{N}$, respectively) at the initial time instant can be obtained from the generating operator $\underline{\underline{R}}(J)$ as follows:

$$
\left.\check{\rho}_{\{m\}_{M} ;\{n\}_{N}}=\left.\frac{\partial^{M+N} \underline{\check{R}}(J)}{\partial\left(J_{m_{1}}^{(+)} \ldots J_{m_{M}}^{(+)} J_{n_{1}}^{(-)} \ldots J_{n_{N}}^{(-)}\right)}\right|_{J=0}=\mid \Psi_{\{m\}_{M} ;\{n\}_{N}}(\text { in })\right\rangle\left\langle\Psi_{\{m\}_{M} ;\{n\}_{N}}(\text { in })\right|,
$$

where

$$
\begin{aligned}
& \left|\Psi_{\{m\}_{M} ;\{n\}_{N}}(i n)\right\rangle=\prod_{i=1}^{M} a_{m_{i}}^{\dagger}(i n) \prod_{j=1}^{N} b_{n_{j}}^{\dagger}(i n)|0, i n\rangle, \\
& \left\langle\Psi_{\{m\}_{M} ;\{n\}_{N}}(i n)\right|=\langle 0, i n| \prod_{j=1}^{N} b_{n_{j}}(i n) \prod_{i=1}^{M} a_{m_{i}}(i n) .
\end{aligned}
$$

c) Let us set

$$
J_{n}^{(\zeta)}=e^{-E_{n}^{(\zeta)}}, E_{n}^{(\zeta)}=\beta\left(\varepsilon_{n}^{(\zeta)}-\mu^{(\zeta)}\right), \beta^{-1}=\Theta
$$

where $\varepsilon_{n}^{(\zeta)}$ are the energies of particles or antiparticles with the quantum numbers $n$; $\mu^{(\zeta)}$ are the corresponding chemical potentials, and $\Theta$ is the absolute temperature. One can see that with such a choice of sources the generating operator (21) becomes the density operator $\check{\rho}_{\beta}$ of the system that has been in thermal equilibrium at the initial time instant. Using relation (105) from Appendix, we obtain an explicit expression for $\check{\rho}_{\beta}$, namely,

$$
\begin{aligned}
& \check{\rho}_{\beta}=\check{R}\left(e^{-E_{n}^{(\zeta)}}\right)=\frac{1}{Z} \exp \left\{-\left[a^{\dagger}(i n) E^{(+)} a(i n)+b^{\dagger}(i n) E^{(-)} b(i n)\right]\right\}, \\
& Z=\exp \left\{\kappa \sum_{n} \ln \left(1+\kappa e^{-E_{n}^{(+)}}\right)+\kappa \sum_{m} \ln \left(1+\kappa e^{-E_{n}^{(-)}}\right)\right\},
\end{aligned}
$$

or

$$
\check{\rho}_{\beta}=Z^{-1} \exp \left\{-\beta\left[\check{H}-\sum_{\zeta= \pm} \mu^{(\zeta)} \check{N}^{(\zeta)}\right]\right\}
$$

where $\check{H}$ is the Hamiltonian of the system (written in terms of $i n$-operators); $\check{N}^{(\zeta)}$ are the operators of in-particle or in-antiparticle numbers,

$$
\begin{aligned}
& \check{H}=a^{\dagger}(i n) \varepsilon^{(+)} a(i n)+b^{\dagger}(i n) \varepsilon^{(-)} b(i n), \\
& \check{N}^{(+)}=a^{\dagger}(i n) a(i n), \check{N}^{(-)}=b^{\dagger}(i n) b(i n),
\end{aligned}
$$

and the matrices $E^{(\zeta)}$ and $\varepsilon^{(\zeta)}$ are defines as $E_{m n}^{(\zeta)}=\delta_{m n} E_{n}^{(\zeta)}$ and $\varepsilon_{m n}^{(\zeta)}=\delta_{m n} \varepsilon_{n}^{(\zeta)}$. 
We can see that the problem of calculating the mean value of an operator $\hat{F}\left(t_{\text {out }}\right)$ for the system being at the final time instant is related to the problem of calculating the quantity $\operatorname{tr}[\check{F} \underline{\check{R}}(J)]$, where $\check{F}$ is a Heisenberg operator corresponding to $\hat{F}\left(t_{\text {out }}\right)$. Such a quantity can be represented as follows:

$$
\begin{aligned}
& \operatorname{tr}[\check{F} \underline{\check{R}}(J)]=\sum_{M, N=0}^{\infty} \sum_{\{m\}\{n\}} \frac{1}{M ! N !}\left\langle\Psi\left(\{m\}_{M},\{n\}_{N}\right)|\check{F} \underline{\underline{R}}(J)| \Psi\left(\{m\}_{M},\{n\}_{N}\right)\right\rangle, \\
& \left|\Psi\left(\{m\}_{M},\{n\}_{N}\right)\right\rangle=a_{m_{1}}^{\dagger} \ldots a_{m_{M}}^{\dagger} b_{n_{1}}^{\dagger} \ldots b_{n_{N}}^{\dagger}|0\rangle \\
& \left\langle\Psi\left(\{m\}_{M},\{n\}_{N}\right)\right|=\langle 0| b_{n_{N}} \ldots b_{n_{1}} a_{m_{M}} \ldots a_{m_{1}}
\end{aligned}
$$

Calculating $\operatorname{tr}[\check{F} \underline{\underline{L}}(J)]$ according to (29), it is convenient to have an expression for the operator $\underline{\check{L}}(J)$ in terms of out-operators. One can see that such an expression has the form

$$
\underline{\underline{R}}(J)=V U(J) V^{\dagger}, \quad U(J)=: \exp \left[a^{\dagger}\left(\mathbb{J}^{(+)}-1\right) a+b^{\dagger}\left(\mathbb{J}^{(-)}-1\right) b\right]:,
$$

where : $\cdots:$ is the sign of the normal form with respect to the out-vacuum, and the operator $V$ is defined by (6). A normal form of the operator $\underline{\check{L}}(J)$ with respect to the out-vacuum is calculated below.

\subsection{The normal form of the generating operator}

First of all, we rewrite the operator expression (30) as follows:

$$
\underline{\check{R}}(J)=v_{4} v_{3} v_{2} \tilde{Y}(J) v_{2}^{\dagger} v_{3}^{\dagger} v_{4}^{\dagger}, \quad \tilde{Y}=v_{1} U(J) v_{1}^{\dagger},
$$

where the operators $\nu_{i}, i=1, \ldots, 4$, are given by (7). Using formula (104) from Appendix, we represent the operator $\tilde{Y}(J)$ in the form

$$
\begin{aligned}
& \tilde{Y}(J)=Y(J) U(J), Y(J)=\exp (-b B a) \exp \left(-a^{\dagger} A(J) b^{\dagger}\right), \\
& A(J)=\mathbb{J}^{(+)} B^{\dagger} \mathbb{J}^{(-)}, B=\kappa w(0 \mid-+) .
\end{aligned}
$$

Both operator exponents in the expression for $Y(J)$ can be written in terms of Gaussian path integrals.

Consider, first of all, the fermi-particle case. In this case, we can treat the anticommuting operators $a$ and $b$ (or $a^{+}$and $b^{+}$) as Grassmann-odd variables. Then, according to representation (22) at $\kappa=1$, we have

$$
\begin{aligned}
& Y=\operatorname{det} A \operatorname{det} B \int \exp \left(\tilde{\lambda}^{*} B^{-1} \tilde{\lambda}+\lambda^{*} A^{-1} \lambda\right) \Phi \Pi d \tilde{\lambda}^{*} d \tilde{\lambda} d \lambda^{*} d \lambda, \\
& \Phi=\exp \left(b \tilde{\lambda}+\tilde{\lambda}^{*} a\right) \exp \left(a^{\dagger} \lambda+\lambda^{*} b^{\dagger}\right) .
\end{aligned}
$$

With the help of relation (106) from Appendix, we represent the operator $\Phi$ in the normal form

$$
\Phi=: \exp \left(a^{\dagger} \lambda+\lambda^{*} b^{\dagger}+b \tilde{\lambda}+\tilde{\lambda}^{*} a+\tilde{\lambda}^{*} \lambda+\tilde{\lambda} \lambda^{*}\right): .
$$

Then, using formula (22) we can calculate the complete path integral (33). In the Bose case, we can examine all the operators $a^{\dagger}, b^{\dagger}, a$, and $b$ as bosonic (ordinary) variables under the normal form sign, so that the operator $Y(J)$ can be represented as a usual Gaussian path integral by applying representation (22) at $\kappa=-1$. Calculating these Gaussian integrals, we obtain the normal form of the operator $Y$,

$$
\begin{aligned}
& Y=\operatorname{det}(1+\kappa A B)^{\kappa}: \exp \left\{-a^{\dagger} A_{++} a-b^{\dagger} A_{--} b-a^{\dagger} A_{+-} b^{\dagger}-b A_{-+} a\right\}:, \\
& A_{++}=\kappa A B(1+\kappa A B)^{-1}, A_{--}^{T}=\kappa B A(1+\kappa B A)^{-1}, \\
& A_{+-}=(1+\kappa A B)^{-1} A, A_{-+}=B(1+\kappa A B)^{-1} .
\end{aligned}
$$

Using relation (107) from Appendix, we represent the operator $\tilde{Y}$ in the normal form

$$
\begin{aligned}
& \tilde{Y}=\operatorname{det}(1+\kappa A B)^{\kappa}: \exp \left\{-a^{\dagger} \widetilde{A}_{++} a-b^{\dagger} \widetilde{A}_{--} b-a^{\dagger} \widetilde{A}_{+-} b^{\dagger}-b \widetilde{A}_{-+} a\right\}:, \\
& \widetilde{A}_{++}=1-\left(1-A_{++}\right) \mathbb{J}^{(+)}, \widetilde{A}_{--}=1-\left(1-A_{--}\right) \mathbb{J}^{(-)}, \\
& \widetilde{A}_{+-}=\widetilde{A}_{-+}^{\dagger}, \widetilde{A}_{-+}=\mathbb{J}^{(-)} A_{-+} \mathbb{J}^{(+)} .
\end{aligned}
$$


With the help of relation (8), we rewrite the operator $v_{3}$ as follows:

$$
v_{3}=\exp \left[-\kappa b \ln w(-\mid-) b^{\dagger}\right]=c_{v} \exp \left[b^{\dagger} \ln w(-\mid-)^{T} b\right] .
$$

Then, using formulas (105) derived in Appendix, we represent the operators $v_{3} v_{2}$ and $v_{2}^{\dagger} v_{3}^{\dagger}$ from (31) in the normal form as follows:

$$
\begin{aligned}
& v_{3} v_{2}=c_{v}: \exp \left[b^{\dagger}\left(w(-\mid-)^{T}-1\right) b\right] \exp \left[a^{\dagger}(w(+\mid+)-1) a\right]:, \\
& v_{2}^{\dagger} v_{3}^{\dagger}=c_{v}^{*}: \exp \left[a^{\dagger}\left(w(+\mid+)^{\dagger}-1\right) a\right] \exp \left[b^{\dagger}\left(w(-\mid-)^{T \dagger}-1\right) b\right]: .
\end{aligned}
$$

Finally, using relation (107), we obtain the normal form of the operator $\underline{\check{L}}(J)$,

$$
\begin{aligned}
& \underline{\check{R}}(J)=\left|c_{v}\right|^{2} \operatorname{det}(1+\kappa A B)^{\kappa}: \exp \left[-a^{\dagger}\left(1-D_{+}\right) a-b^{\dagger}\left(1-D_{-}\right) b-a^{\dagger} C^{\dagger} b^{\dagger}-b C a\right]:, \\
& D_{+}=w(+\mid+)(1+\kappa A B)^{-1} \mathbb{J}^{(+)} w(+\mid+)^{\dagger}, \\
& D_{-}^{T}=w(-\mid-)^{\dagger} \mathbb{J}^{(-)}(1+\kappa B A)^{-1} w(-\mid-), \\
& C=w(-\mid-)^{\dagger} \mathbb{J}^{(-)} B(1+\kappa A B)^{-1} \mathbb{J}^{(+)} w(+\mid+)^{\dagger}+\kappa w(+-\mid 0)^{\dagger} .
\end{aligned}
$$

Representation (36) is useful, since it allows one to calculate the trace (29) by using the path integral techniques described in Appendix; see, eq. (110).

As an example, let us consider the density operator $\check{\rho}_{v}$ defined by (24). Using (36), we represent this operator in terms of out-operators, as well as in the normal form

$$
\check{\rho}_{v}=\underline{\check{R}}(0)=\left|c_{v}\right|^{2}: \exp \left[-a^{\dagger} a-b^{\dagger} b-\kappa a^{\dagger} w(+-\mid 0) b^{\dagger}-\kappa b w(+-\mid 0)^{\dagger} a\right]: .
$$

In a similar way, the operator $\check{\phi}(J)$ in the expression of generating functional (25) can be transformed to the normal form

$$
\check{\phi}(J)=\left|c_{v}\right|^{2}: \exp \left[-a^{\dagger} a-b^{\dagger} b-\kappa a^{\dagger} e^{\mathbb{J}^{(+)}} w(+-\mid 0) e^{\mathbb{J}^{(-)}} b^{\dagger}-\kappa b w(+-\mid 0)^{\dagger} a\right]:,
$$

where the representation (37) for $\check{\rho}_{v}$ has been used. Then, applying the path integral representation for traces (110), and using formula (107), we represent the generating functional of momenta as follows:

$$
\Phi^{v}(J)=\left|c_{v}\right|^{2} \exp \left\{\kappa \operatorname{tr} \ln \left[1+\kappa w(+-\mid 0)^{\dagger} e^{\mathbb{J}^{(+)}} w(+-\mid 0) e^{\mathbb{J}^{(-)}}\right]\right\}
$$

\section{Reduced density operators and correlation functions}

\subsection{Reduced density operators}

In the general case, the states of the system under consideration at the final time instant contain both particles and antiparticles due to the pair-creation by external fields and the structure of the initial state. On the other hand, we are often interested in physical quantities $F_{ \pm}$which describe only particles $(+)$or antiparticles $(-)$ at the final time instant. The corresponding operators $\check{F}_{ \pm}$are functions of either $a^{\dagger}, a$ or $b^{\dagger}, b$,

$$
\check{F}_{+}=F_{+}\left(a^{\dagger}, a\right), \check{F}_{-}=F_{-}\left(b^{\dagger}, b\right) .
$$

The mean values of operators $\check{F}_{ \pm}$and the entire information concerning the subsystems of particles and antiparticles can be obtained from the so-called reduced density operators, which we shall define below.

We present the basis vectors from (29) as follows:

$$
\begin{aligned}
& \left|\Psi\left(\{m\}_{M},\{n\}_{N}\right)\right\rangle=\left|\Psi_{a}\left(\{m\}_{M}\right)\right\rangle \otimes\left|\Psi_{b}\left(\{n\}_{N}\right)\right\rangle,|0\rangle=|0\rangle_{a} \otimes|0\rangle_{b}, \\
& \left|\Psi_{a}\left(\{m\}_{M}\right)\right\rangle=a_{m_{1}}^{\dagger} \ldots a_{m_{M}}^{\dagger}|0\rangle_{a},\left|\Psi_{b}\left(\{n\}_{N}\right)\right\rangle=b_{n_{1}}^{\dagger} \ldots b_{n_{N}}^{\dagger}|0\rangle_{b},
\end{aligned}
$$

where $|0\rangle_{a}$ and $|0\rangle_{b}$ are the vacuum vectors of particle and antiparticle subsystems. The mean values of the operators $\check{F}_{ \pm}$are

$$
\left\langle F_{ \pm}\right\rangle=\operatorname{tr}_{+} \operatorname{tr}_{-}\left(\check{F}_{ \pm} \check{\rho}\right),
$$


where $\check{\rho}$ is the density operator of the system, and the reduced traces $\operatorname{tr}_{ \pm}$of an operator $\check{A}$ are defined as

$$
\begin{aligned}
& \operatorname{tr}_{+} \check{A}=\sum_{M=0}^{\infty} \sum_{\{m\}}(M !)^{-1}\left\langle\Psi_{a}\left(\{m\}_{M}\right)|\check{A}| \Psi_{a}\left(\{m\}_{M}\right)\right\rangle, \\
& \operatorname{tr}_{-} \check{A}=\sum_{M=0}^{\infty} \sum_{\{m\}}(M !)^{-1}\left\langle\Psi_{b}\left(\{m\}_{M}\right)|\check{A}| \Psi_{b}\left(\{m\}_{M}\right)\right\rangle .
\end{aligned}
$$

We define the reduced density operators (in the Heisenberg picture) $\check{\rho}_{ \pm}$of the subsystems of particles and antiparticles, respectively, as follows:

$$
\check{\rho}_{ \pm}=\operatorname{tr}_{\mp} \check{\rho} .
$$

Then mean values (42) can be calculated with the help of the reduced density operators $\check{\rho}_{ \pm}$as follows:

$$
\left\langle F_{ \pm}\right\rangle=\operatorname{tr}_{ \pm}\left(\check{F}_{ \pm} \check{\rho}_{ \pm}\right) \text {. }
$$

Even if the initial state of the system is a pure state, the reduced density operators $\check{\rho}_{ \pm}$describe mixed states. In some physical problems, the use of a reduced density operators is inevitable. For example, considering particle creation by the gravitation field of a black hole we have only the reduced operator of the particles created outside the black hole, since we do not have any information about the particles behind the horizon, [8, 9].

In a similar manner, we introduce the reduced generating operators $\check{R}_{ \pm}(J)$ as follows:

$$
\check{R}_{ \pm}(J)=\operatorname{tr}_{\mp} \check{R}(J) .
$$

Using the path integral representation for traces (110), representation (36), as well as (107), we obtain

$$
\begin{aligned}
& \check{R}_{+}(J)=Z_{+}^{-1}: \exp \left\{-a^{\dagger}\left(1-K_{+}(J)\right) a\right\}:, \\
& \check{R}_{-}(J)=Z_{-}^{-1}: \exp \left\{-b^{\dagger}\left(1-K_{-}(J)\right) b\right\}:, \\
& K_{ \pm}(J)=D_{ \pm}+C^{\dagger}\left(1+\kappa D_{\mp}^{T}\right)^{-\kappa} C, \\
& Z_{ \pm}^{-1}(J)=Z^{-1}\left|c_{v}\right|^{2} \operatorname{det}(1+\kappa A B)^{\kappa} \operatorname{det}\left(1+\kappa D_{\mp}\right)^{\kappa} .
\end{aligned}
$$

The reduced generating operators $\check{R}_{ \pm}(J)$ allow one to obtain the reduced density operators $\check{\rho}_{ \pm}$for different initial states of the system. Consider below some examples:

a) Selecting all $J=0$ in (46), we obtain reduced density operators $\check{\rho}_{v \pm}=\check{R}_{ \pm}(0)$ of a system that has been in a pure vacuum state at the initial time instant. Explicit expressions for $\check{R}_{ \pm}(0)$ follow from (46) with account taken of

$$
K_{ \pm}(0)=w(+-\mid 0) w(+-\mid 0)^{\dagger}, Z_{ \pm}^{-1}(0)=\left|c_{v}\right|^{2} .
$$

The same result has been obtained in [24, 34] by a straightforward calculation.

b) The reduced density operators $\check{\rho}_{0 ; n \pm}$ and $\check{\rho}_{m ; 0 \pm}$ of a system that has been in a pure state with particles or antiparticles, respectively, at the initial time instant can be obtained from the generating operator $\underline{\check{R}}_{ \pm}(J)=Z \check{R}_{ \pm}(J)$ as follows:

$$
\begin{aligned}
& \check{\rho}_{m ; 0+}=\left.\frac{\partial \underline{\underline{R}}_{+}(J)}{\partial J_{m}^{(+)}}\right|_{J=0}=\left[a^{\dagger} w(+\mid+)\right]_{m} \check{\rho}_{v+}\left[w(+\mid+)^{\dagger} a\right]_{m}, \\
& \check{\rho}_{0 ; m-}=\left.\frac{\partial \underline{\underline{R}}_{-}(J)}{\partial J_{m}^{(-)}}\right|_{J=0}=\left[w(-\mid-) b^{\dagger}\right]_{m} \check{\rho}_{v-}\left[b w(-\mid-)^{\dagger}\right]_{m}, \\
& \check{\rho}_{0 ; m+}=\left.\frac{\partial \underline{\underline{R}}_{+}(J)}{\partial J_{m}^{(-)}}\right|_{J=0}=\check{\rho}_{v+}\left[w(-\mid-) w(-\mid-)^{\dagger}\right]_{m m} \\
& -\left[a^{\dagger} w(+-\mid 0) w(-\mid-)^{\dagger}\right]_{m} \check{\rho}_{v+}\left[w(-\mid-) w(+-\mid 0)^{\dagger} a\right]_{m}, \\
& \check{\rho}_{m ; 0-}=\left.\frac{\partial \underline{\underline{R}}_{-}(J)}{\partial J_{m}^{(+)}}\right|_{J=0}=\check{\rho}_{v-}\left[w(+\mid+)^{\dagger} w(+\mid+)\right]_{m m} \\
& -\left[b^{\dagger} w(+-\mid 0) w(+\mid+)^{*}\right]_{m} \check{\rho}_{v-}\left[w(+\mid+)^{T} w(+-\mid 0)^{\dagger} b\right]_{m} .
\end{aligned}
$$

\footnotetext{
${ }^{4}$ It should be noted that the symbols of the normal form of the operator $\hat{R}_{\zeta}$ have been presented in 24 via some path integrals. The explicit form of operator has been presented for $J_{n}^{(\zeta)}=e^{-E_{n}^{(\zeta)}}$. Unfortunately, it contains some misprints.
} 
c) Let us set the sources in (46) as in (27). One can see that for such a choice of sources the reduced generating operators (21) become the reduced density operators $\check{\rho}_{\beta \pm}$ of the system that has been in thermal equilibrium at the initial time instant.

\subsection{One-particle correlation functions}

Let us now examine the following generating functions:

$$
\begin{aligned}
& \mathbb{N}_{n m}^{(+)}=\operatorname{tr}\left(a_{n}^{\dagger} a_{m} \check{R}\right)=\operatorname{tr}_{+}\left(a_{n}^{\dagger} a_{m} \check{R}_{+}\right), \\
& \mathbb{N}_{n m}^{(-)}=\operatorname{tr}\left(b_{n}^{\dagger} b_{m} \check{R}\right)=\operatorname{tr}_{-}\left(b_{n}^{\dagger} b_{m} \check{R}_{+}\right) .
\end{aligned}
$$

They generate one-particle correlation functions for different initial states of the system. Setting the sources (taking the corresponding derivatives, if necessary) in (48) as has been demonstrated in Sec. 2, we choose the required initial states. The diagonal elements $\mathbb{N}_{m m}^{(\zeta)}$ are the generating functionals for the mean numbers $N_{m}^{(\zeta)}$ of particles/antiparticles with quantum numbers $m$ at the final time instant (further differential mean numbers). In what follows, we refer to quantities (48) as correlation functions.

The correlation functions $\mathbb{N}_{n m}^{(\zeta)}$ can be expressed via the matrices $K_{\zeta}$ (46), and vice-versa, as follows:

$$
\mathbb{N}^{(\zeta)}=\left(\frac{K_{\zeta}}{1+\kappa K_{\zeta}}\right)^{T}, K_{\zeta}=\frac{\mathbb{N}^{(\zeta) T}}{1-\kappa \mathbb{N}^{(\zeta) T}}
$$

Note that the quantities $K_{\zeta}$ are functions of elementary probability amplitudes (4).

Relations (49) can be proved as follows: first, using the commutation relations (1) we represent (48) as traces of operators in the normal form:

$$
\mathbb{N}_{n m}^{(+)}=\operatorname{tr}_{+}\left[a_{n}^{\dagger} \check{R}_{+}\left(K_{+} a\right)_{m}\right], \quad \mathbb{N}_{n m}^{(-)}=\operatorname{tr}_{-}\left[b_{n}^{\dagger} \check{R}_{-}\left(K_{-} b\right)_{m}\right] .
$$

The quantities $\mathbb{N}_{n m}^{(\zeta)}$ can be obtained from the generating functions $\mathcal{Z}_{\zeta}(\bar{j}, j)$ as follows:

$$
\mathbb{N}_{n m}^{(\zeta)}=\left.\frac{\partial^{2} \mathcal{Z}_{\zeta}(\bar{j}, j)}{\partial \bar{j}_{n} \partial j_{m}}\right|_{\bar{j}=j=0}
$$

where

$$
\begin{aligned}
& \mathcal{Z}_{+}(\bar{j}, j)=Z_{+}^{-1} \operatorname{tr}_{+}: \exp \left\{-a^{\dagger}\left[1-\mathbb{I} K_{+}\right] a\right\}:, \\
& \mathcal{Z}_{-}(\bar{j}, j)=Z_{-}^{-1} \operatorname{tr}_{-}: \exp \left\{-b^{\dagger}\left[1-\mathbb{I} K_{-}\right] b\right\}:, \\
& \mathcal{Z}_{\zeta}(0,0)=1, \mathbb{I}_{m n}=\delta_{m n}+\bar{j}_{m} j_{n}, \zeta= \pm
\end{aligned}
$$

and $\bar{j}$ and $j$ are some new sources. Traces in (52) can be calculated by formula (110) from Appendix. Thus, we obtain

$$
\mathcal{Z}_{\zeta}(\bar{j}, j)=Z_{\zeta}^{-1} \exp \left\{\kappa \sum_{n}\left[\ln \left(1+\kappa \mathbb{I} K_{\zeta}\right)\right]_{n n}\right\} .
$$

Then, relations (49) follow from (51) and (53).

The normalization conditions and the second relation (49) imply that the quantities $Z_{\zeta}$ can be expressed in terms of $\mathbb{N}^{(\zeta)}$ as

$$
Z_{\zeta}=\exp \left\{\kappa \sum_{n}\left[\ln \left(1+\kappa K_{\zeta}\right)\right]_{n n}\right\}=\exp \left\{-\kappa \sum_{n}\left[\ln \left(1-\kappa \mathbb{N}^{(\zeta) T}\right)\right]_{n n}\right\} .
$$

Now, we are going to relate the quantities $\mathbb{N}_{n m}^{(\zeta)}$ with the correlation functions $\mathbb{N}_{n m}^{(\zeta)}(i n)$ of $i n$-operators,

$$
\mathbb{N}_{n m}^{(+)}(i n)=\operatorname{tr}\left[a_{n}^{\dagger}(i n) a_{m}(i n) \check{R}\right], \mathbb{N}_{n m}^{(-)}(i n)=\operatorname{tr}\left[b_{n}^{\dagger}(i n) b_{m}(i n) \check{R}\right] .
$$

Using representation (21) for $\check{R}$, one can see that

$$
\mathbb{N}_{n m}^{(\zeta)}(i n)=\delta_{n m} N_{m}^{(\zeta)}(i n), \quad N_{m}^{(\zeta)}(i n)=\frac{J_{m}^{(\zeta)}}{1+\kappa J_{m}^{(\zeta)}},
$$


where $N_{m}^{(\zeta)}(i n)$ are the differential mean numbers (generating functions for differential mean numbers). Indeed, let us take expressions (48) for $\mathbb{N}^{(\zeta)}$ via traces in the complete Fock space. These traces can be written in the $i n$-basis $\left|\Psi\left(\{m\}_{M},\{n\}_{N} ; i n\right)\right\rangle=V\left|\Psi\left(\{m\}_{M},\{n\}_{N}\right)\right\rangle$. Using the canonical transformation (17), we express the operators $a^{\dagger}, a, b^{\dagger}, b$ via the operators $a^{\dagger}(i n), a(i n), b^{\dagger}(i n), b(i n)$ and calculate the traces explicitly. Then we obtain

$$
\begin{aligned}
& \mathbb{N}^{(+) T}=G\left(\left.{ }^{+}\right|_{+}\right) \mathbb{N}^{(+)}(i n) G\left(\left.{ }_{+}\right|^{+}\right)+G\left(\left.{ }^{+}\right|_{-}\right)\left[1-\kappa \mathbb{N}^{(-)}(i n)\right] G\left(\left.{ }_{-}\right|^{+}\right), \\
& \mathbb{N}^{(-)}=G\left(\left.{ }^{-}\right|_{-}\right) \mathbb{N}^{(-)}(i n) G\left(\left.{ }_{-}\right|^{-}\right)+G\left(\left.{ }^{-}\right|_{+}\right)\left[1-\kappa \mathbb{N}^{(+)}(i n)\right] G\left(\left.{ }_{+}\right|^{-}\right) .
\end{aligned}
$$

Thus, due to relations (49), (57), we have explicit expressions for the complete generating density operator (36) and reduced generating density operators (46) via both correlation functions of $i n$-particles and out-particles, and via elementary probability amplitudes (4) as well.

We stress that there is a one-to-one correspondence between the one-particle correlation functions, and the form of the reduced density operator of the complete system. This correspondence is related to the choice of a model, which is a quantized field placed in an external background. In fact, we deal with a quadratic system of noninteracting (between themselves) particles. Of course, this fact is well-known for free-particle systems. Our consideration generalizes this desription to the presence of a particle-creating background. For systems of interacting particles, there remains an important question: suppose the one-particle distribution at the final time instant is a thermal one. Can one assert that the complete system is in a thermal state with a given temperature (one that determines a one-particle distribution)? Such a question seems to be relevant to the problem of particle creation by black-hole gravitational fields (Hawking's radiation), where one-particle distributions of created particles have a thermal form.

Below, we examine some illustrations of the previously obtained general formulas.

Let the initial state of the system be vacuum $(J=0)$, then (57) reproduces formulas (19) for the differential mean numbers $\aleph_{m}^{(\zeta)}=\left.N_{m}^{(\zeta)}\right|_{J=0}$ of particles/antiparticles created from vacuum by an external field.

Let us examine a common case (for example, a uniform external field) when particle/antiparticle states are specified by quantum numbers (the same being valid for particles and antiparticles) that are integrals of motion. In this case, all the matrices $G\left(\zeta_{\zeta} \zeta^{\prime}\right)$ in (15) are diagonal and the differential mean numbers (19) of particles/antiparticles created from vacuum coincide: $\aleph_{m}^{(+)}=\aleph_{m}^{(-)}=\aleph_{m}$. Using formulas (57), (19), and the unitarity relations (16), one can obtain the expressions for the differential mean numbers of particles/antiparticles, namely,

$$
N_{m}^{(\zeta)}=\left(1-\kappa \aleph_{m}\right) N_{m}^{(\zeta)}(i n)+\aleph_{m}\left[1-\kappa N_{m}^{(-\zeta)}(i n)\right] .
$$

If the initial state differs from vacuum, the differential mean numbers of particles/antiparticles created by the external field are given by the difference $\Delta N_{m}^{(\zeta)}=N_{m}^{(\zeta)}-N_{m}^{(\zeta)}(i n)$. One can see that

$$
\begin{aligned}
& \Delta N_{m}^{(+)}=\Delta N_{m}^{(-)}=\Delta N_{m}, \\
& \Delta N_{m}=\aleph_{m}\left[1-\kappa\left(N_{m}^{(+)}(i n)+N_{m}^{(-)}(i n)\right)\right] .
\end{aligned}
$$

Even if $\aleph_{m} \neq 0$, no particle creation of fermions with quantum numbers $m$ occurs if $N_{m}^{(+)}(i n)+N_{m}^{(-)}(i n)=$ 1. Since $\kappa=-1$ for bosons, $\Delta N_{m}$ is always positive and is larger than $\aleph_{m}$. That is, the presence of matter at the initial state increases the mean number of created bosons.

In some articles devoted to the chromoelectric flux tube model (see, e.g., [12, 14, 35]), one encounters an (inexact) interpretation of the well-known Schwinger formulas describing pair-creation from vacuum by a constant electric field [2]. This interpretation may lead to incorrect results for some field strengths, as noted in [36. Below, we discuss this problem and present correct relations that will be used in the subsequent section. We recall that, by using the proper-time method, Schwinger calculated the one-loop effective Lagrangian $L$ in electric field and assumed that the probability $P^{v}$ of no actual paircreation occurring in the history of the field during the time $T$ in the volume $V$ can be presented as $P^{v}=\left|c_{v}\right|^{2}=\exp \{-V T 2 \operatorname{Im} L\}$ (for a subsequent development, see the review [37). Schwinger interpreted $2 \operatorname{Im} L$ as the probability, per time unit, and per volume unit, of creating a pair by a constant electric field. Some arguments in favour of such an interpretation can be found, for example, in the 
book [38] and the article [15. The interpretation remains approximately valid as long as the WKB calculation is applicable, that is, $V T 2 \operatorname{Im} L \ll 1$. Then the total probability of pair-creation reads as $1-P^{v} \approx V T 2 \operatorname{Im} L$. To calculate the differential probabilities of pair-creation with quantum numbers $m$ (for instance, momentum and spin polarization), one can represent the probability $P^{v}$ as an infinite product:

$$
P^{v}=\prod_{m} e^{-2 \operatorname{Im} S_{m}},
$$

where a certain discretization scheme is used, so that the effective action $S=V T L$ is written as $S=$ $\sum_{m} S_{m}$. All this is possible only if $m$ are selected as integrals of motion. Then, $e^{-2 \operatorname{Im} S_{m}}$ is the vacuumpersistence probability in a cell of the space of quantum numbers $m$. Using the WKB approximation in the case $2 \operatorname{Im} S_{m} \ll 1$, one obtains for the probability $P_{m}$ of a single pair-production with quantum numbers $m$ and for the corresponding mean values $\aleph_{m}$ of created pairs the following relation:

$$
\aleph_{m} \approx P_{m} \approx 2 \operatorname{Im} S_{m}
$$

By analogy with one-particle quantum mechanics, one usually rewrites (61) for fermions,

$$
\aleph_{m} \approx-\ln \left(1-P_{m}\right) \approx 2 \operatorname{Im} S_{m} .
$$

It is clear that (61) and (62) coincide in the first order with respect to $P_{m}$. Then, it follows from (60) that

$$
P^{v} \approx \prod_{m}\left(1-P_{m}\right) .
$$

Using the same analogy for bosons and rewriting (61) as

$$
\aleph_{m} \approx \ln \left(1+P_{m}\right) \approx 2 \operatorname{Im} S_{m},
$$

one obtains the following approximate relation:

$$
P^{v} \approx \prod_{m}\left(1+P_{m}\right)^{-1} .
$$

It turns out that for the field under consideration, by using the WKB calculations and relations (62)(65), one can reproduce Schwinger's result for $P^{v}$. This fact brings the temptation to interpret the latter formulas as exact ones, replacing there " $\approx$ " by " $=$ ". However, one should say that such an interpretation is, in particular, equivalent to the assumption $\aleph_{m}=2 \operatorname{Im} S_{m}$. Nevertheless, as we shall demonstrate below, the latter relation is not an exact one and it is valid only in the approximation $2 \operatorname{Im} S_{m} \ll 1$.

An exact treatment in the framework of QFT with unstable vacuum (see, for example, [4, 28, 30]) yields the following expressions for the scattering $P(-\mid-)_{m}$ of a particle (and an antiparticle) and paircreation $P(+-\mid 0)_{m}$ probabilities, respectively (see Subsection 2.1 for notation):

$$
P(-\mid-)_{m}=\left|w(-\mid-)_{m m}\right|^{2} P^{v}, \quad P(+-\mid 0)_{m}=\left|w(+-\mid 0)_{m m}\right|^{2} P^{v},
$$

where, due to relations (18), (19) and (16), the corresponding relative probabilities are

$$
\left|w(-\mid-)_{m m}\right|^{2}=\frac{1}{1-\kappa \aleph_{m}},\left|w(+-\mid 0)_{m m}\right|^{2}=\frac{\aleph_{m}}{1-\kappa \aleph_{m}} .
$$

As long as the semiclassical approximation is concerned $\left(P^{v} \approx 1, \aleph_{m} \ll 1\right)$, we have

$$
P(+-\mid 0)_{m} \approx\left|w(+-\mid 0)_{m m}\right|^{2} \approx \aleph_{m} .
$$

Thus, we can see that the quantities $P(+-\mid 0)_{m},\left|w(+-\mid 0)_{m m}\right|^{2}$ and $\aleph_{m}$ can be identified only in the approximation under consideration. An exact expression for $P^{v}$ in terms of the mean values $\aleph_{m}$ follows from (8), (67) and reads

$$
P^{v}=\exp \left\{\kappa \sum_{m} \ln \left(1-\kappa \aleph_{m}\right)\right\} .
$$

Formulas (60) and (68) imply the following exact relation between $\operatorname{Im} S_{m}$ and $\aleph_{m}$ :

$$
2 \operatorname{Im} S_{m}=-\kappa \ln \left(1-\kappa \aleph_{m}\right) .
$$

It has to be used in the general case when the WKB approximation is not applicable. 


\subsection{Is it really a thermal distribution?}

Considerable attention has been recently focused on a mechanism of fast thermalization in heavy-ion collisions (see [14, 39] and references therein). A possibility is discussed of a thermal one-particle distribution due to quantum creation of particles from vacuum by strong electric-like fields. Some of these distributions are known in QED, and their relation to thermal spectrum of Hawking's radiation has been discussed (see references in the next section). We give some examples of such distributions in Subsection 4.3. However, a thermal one-particle distribution of created particles does not guarantee the character of thermal equilibrium for the corresponding complete quantum state of the system and only mimics, in some sense, the latter state.

One ought to say that, in contrast to the case of Hawking's radiation, in which we do not have any information about another member of each created pair behind the horizon, both particles and antiparticles created from vacuum by chromoelectric field can, in principle, be observed. Because of the one-to-one correspondence between one-particle correlation functions and the reduced density operator of the complete system, all the moments of particle (or antiparticle) distribution coincide. However, the higher moments of the simultaneous distributions of particles and antiparticles are different.

In what follows, we present a formal analysis of the above problems.

Suppose that the differential mean numbers $N_{m}$ of particles/antiparticles at the final state of a system subject to an external field have the form of a one-particle thermal distribution. There arises the question if one can be sure if in such a case the final state of the complete system is in thermal equilibrium, or the thermal form of a one-particle distribution can appear even if the final state of the complete system is not in thermal equilibrium. To answer these questions, we plan to examine two different possibilities of having the same one-particle thermal distribution for two distinct states of the complete system, one of them being a thermal equilibrium and the other a pure state. Let the first state of the complete system be described by the thermal density operator:

$$
\begin{aligned}
& \check{\rho}_{\beta}^{\text {out }}=\frac{1}{Z} \exp \left\{-\left[a^{\dagger} E^{(+)} a+b^{\dagger} E^{(-)} b\right]\right\}, \\
& Z=\exp \left\{\kappa \sum_{n} \ln \left(1+\kappa e^{-E_{n}^{(+)}}\right)+\kappa \sum_{m} \ln \left(1+\kappa e^{-E_{n}^{(-)}}\right)\right\},
\end{aligned}
$$

where $E^{( \pm)}$are given by (27). It is obvious that in such a state the differential mean numbers $N_{m}$ have the form

$$
N_{m}=\left(e^{E_{m}}+\kappa\right)^{-1} \text {. }
$$

On the other hand, if we have a causal evolution from vacuum, the density operator of the corresponding pure state having the form $\check{\rho}_{v}$ (24); see the normal form in (37). Such a state provides the differential mean numbers (71) in case (67) holds true. We can see that measuring the one-particle distribution cannot distinguish between both these cases. Nevertheless, they can be distinguished by measuring the next moments, as demonstrated below. Let us calculate the variances $\operatorname{Var}_{m}$ in the states described by the density matrices (70) and (24), respectively,

$$
\begin{aligned}
& \operatorname{Var}_{m}^{\text {th }}=\operatorname{tr}\left[\left(a_{m}^{\dagger} a_{m}+b_{m}^{\dagger} b_{m}-2 N_{m}\right)^{2} \check{\rho}_{\beta}^{\text {out }}\right], \\
& \operatorname{Var}_{m}^{v}=\operatorname{tr}\left[\left(a_{m}^{\dagger} a_{m}+b_{m}^{\dagger} b_{m}-2 N_{m}\right)^{2} \check{\rho}_{v}\right] .
\end{aligned}
$$

Since the differential mean values coincide in both states, one can see that

$$
\begin{aligned}
& \operatorname{Var}_{m}^{t h}-\operatorname{Var}_{m}^{v}=2\left(Q_{m}^{t h}-Q_{m}^{v}\right), \\
& Q_{m}^{t h}=\operatorname{tr}\left[a_{m}^{\dagger} a_{m} b_{m}^{\dagger} b_{m} \check{\rho}_{\beta}^{\text {out }}\right], Q_{m}^{v}=\operatorname{tr}\left[a_{m}^{\dagger} a_{m} b_{m}^{\dagger} b_{m} \check{\rho}_{v}\right] .
\end{aligned}
$$

To calculate the quantities $Q_{m}^{t h}, Q_{m}^{v}$ and demonstrate that they are actually different, we are going to use the generating functional $\Phi^{v}(J)$ (39), and the generating functional of momenta for the thermal distribution,

$$
\Phi^{t h}(J)=\operatorname{tr}\left\{\exp \left[a^{\dagger} \mathbb{J}^{(+)} a+b^{\dagger} \mathbb{J}^{(-)} b\right] \check{\rho}_{\beta}^{\text {out }}\right\} .
$$

Then

$$
Q_{m}^{t h}=\left.\frac{\partial^{2} \Phi^{t h}}{\partial J_{m}^{(+)} \partial J_{m}^{(-)}}\right|_{J=0}=N_{m}^{2}
$$


where the expression

$$
\Phi^{t h}(J)=\frac{1}{Z} \exp \left\{\kappa \sum_{n} \ln \left(1+\kappa e^{-E_{n}^{(+)}+J_{n}^{(+)}}\right)+\kappa \sum_{m} \ln \left(1+\kappa e^{-E_{n}^{(-)}+J_{n}^{(-)}}\right)\right\}
$$

is used. On the other hand,

$$
Q_{m}^{v}=\left.\frac{\partial^{2} \Phi^{v}}{\partial J_{m}^{(+)} \partial J_{m}^{(-)}}\right|_{J=0}=N_{m}\left[1+(1-\kappa) N_{m}\right] .
$$

Thus, we can see that the variances of the simultaneous distributions of particles and antiparticles, $\operatorname{Var}_{m}^{t h}$ and $\operatorname{Var}_{m}^{v}$, are quite different:

$$
\operatorname{Var}_{m}^{t h}-\operatorname{Var}_{m}^{v}=2 N_{m}\left(\kappa N_{m}-1\right)
$$

\section{Particle-creation in an electric-like background}

\subsection{Quasi-constant electric field}

Below, we consider a number of applications of the above-developed formalism in QED with a quasiconstant (slowly varying) uniform electric field violating the stability of vacuum. We emphasize that our consideration can be relevant in QCD with an electric-like colour field and in some QFT models with a curved space-time, as was demonstrated, for example, in [40. It was shown [4 that the distribution of pairs created from vacuum by a quasi-constant electric field has a thermal-like form. It appears that such a form has a universal character, i.e., it emerges in any theory with quasi-constant external fields; and when applied to particle-creation in external constant gravitational fields it reproduces exactly the Hawking temperature. Thus, our consideration of QED with a quasi-constant electric field allows one to reveal the typical properties of any strong-field QFT.

Note that in the case under consideration particle states are specified by continuous quantum numbers of the momentum $\mathbf{p}$ and spin projections $r= \pm 1$ (we formally set $r=0$ for scalar particles). From now on, we suppose that the standard volume regularization is used, so that $\delta\left(\mathbf{p}-\mathbf{p}^{\prime}\right)$ is replaced by $\delta_{\mathbf{p}, \mathbf{p}^{\prime}}$ in the normalization conditions. Thus, our particles are labeled by a set of discrete quantum numbers, $m=(\mathbf{p}, r)$.

As usual, we shall describe the electric field by time-dependent vector potentials. The states of the quantum system in question are far from equilibrium due to the field influence. We shall study in detail the time dependence of various mean values, in particular, the mean values of created particles. In a physically correct setting of the problem, we consider a model of a quasi-constant electric field $E\left(x^{0}\right)$ which effectively acts only for a finite period of time $T$ and is zero outside this interval (we further call it the $T$-constant field). In our model, $E\left(x^{0}\right)=E$ for $t_{1} \leq x^{0} \leq t_{2}, t_{2}=-t_{1}=T / 2$. Thus, the field produces finite work in a finite space volume. We accept the initial vacuum to be a free-particle vacuum. A relevant calculation in QED with a $T$-constant field can be found in [4. Below, we use these results for evaluating the leading terms in particle-creation phenomena at large $T$, when the effects of switching on and off are negligible.

Let us describe the $T$-constant field. It is nonstationary but has a constant space direction. We place the latter field along the $x^{3}$-axis. We denote by $q$ the charge of a particle (by $-q$ that of an antiparticle), and by $M$ we denote mass. The corresponding potentials can be chosen in the form: $A_{0}=A_{1}=A_{2}=0$, and

$$
A_{3}\left(x^{0}\right)= \begin{cases}E t_{1}, & x^{0} \in I \\ E x^{0}, & x^{0} \in I I \\ E t_{2}, & x^{0} \in I I I .\end{cases}
$$

where the time intervals are $I=\left(-\infty, t_{1}\right), I I=\left[t_{1}, t_{2}\right], I I I=\left(t_{2},+\infty\right)$.

If the time $T$ is sufficiently large,

$$
T>>T_{0}=(1+\lambda) / \sqrt{|q E|},
$$

the differential mean numbers $\aleph_{m}$ read

$$
\aleph_{m}=\left\{\begin{array}{l}
e^{-\pi \lambda}\left[1+O\left(\left[\frac{1+\lambda}{K}\right]^{3}\right)\right], \quad-\sqrt{|q E|} \frac{T}{2} \leq \xi \leq-K, \\
O(1),-K<\xi \leq+K \\
O\left(\left[\frac{1+\lambda}{\xi^{2}}\right]^{3}\right), \quad \xi>K
\end{array}\right.
$$


where $K$ is a sufficiently large arbitrary constant, $K>>1+\lambda$,

$$
\lambda=\frac{M^{2}+\mathbf{p}_{\perp}^{2}}{|q E|}, \mathbf{p}_{\perp}=\left(p^{1}, p^{2}, 0\right), \quad \xi=\frac{\left|p_{3}\right|-|q E| T / 2}{\sqrt{|q E|}},
$$

and $p_{3}$ is a longitudinal momentum of a particle [4. One can examine the limit $T \rightarrow \infty$ at any given $\mathbf{p}$ in the above expression. In such a limit, the differential mean numbers have a simple form:

$$
\aleph_{m}=e^{-\pi \lambda}
$$

which coincides with that obtained in a constant electric field by Nikishov 3. One can see that the stabilization of the differential mean numbers to the asymptotic form (77) for finite longitudinal momenta is reached at $T \gg T_{0}$. The characteristic time $T_{0}$ is called the stabilization time.

In order to investigate the effects of switching on and off for $T>>T_{0}$, we are going to consider a different example of a quasi-constant electric field:

$$
E\left(x^{0}\right)=E \cosh ^{-2}\left(\frac{x^{0}}{\alpha}\right) .
$$

This field switches on and off adiabatically as $x^{0} \rightarrow \pm \infty$ and is quasi-constant at finite times. It is called an adiabatic field. The differential mean numbers of particles created by such a field have been found in [41. For a further discussion, we need these numbers for a large $\alpha$. As has been demonstrated in [4, the differential mean numbers in the field (78) take the asymptotic form (77) for $\alpha>>\alpha_{0}=(1+\sqrt{\lambda}) / \sqrt{|q E|}$ and for $\left|p_{3}\right|<<|q E| \alpha$. Thus, $\alpha_{0}$ can be interpreted as the stabilization time for an adiabatic field. At the same time, the latter fact means that the effects of switching on and off are not essential at large times and finite longitudinal momenta for both fields. Extrapolating this conclusion, one can suppose that particle-creation effects in any electric field, that is one being quasi-constant $\approx E$ at least for a time period $T>T_{0}$ and switching on and off outside this period arbitrarily, do not depend on the details of switching on and off. Thus, our calculations in a $T$-constant field are typical for a large class of quasi-constant electric fields.

It is of interest for phenomenological applications to calculate the distribution of particles created with all possible $p_{3}$ values and a given $p_{\perp}$ (it is called the $p_{\perp}$ distribution and is denoted by $n_{p_{\perp}}$, in what follows). Analysing the total mean number of particles created by the $T$-constant field, we go over from summation to integration: $\sum_{\mathbf{p}} \rightarrow \frac{V}{(2 \pi)^{3}} \int d \mathbf{p}$. Then, the total mean number (we denote it by $\aleph$ ) can be presented as

$$
\aleph=V \int d^{2} p_{\perp} n_{p_{\perp}}
$$

where

$$
n_{p_{\perp}}=\frac{1}{(2 \pi)^{3}} \sum_{r} \int d p_{3} \aleph_{m}
$$

is the $p_{\perp}$ distribution density of particles created per unit volume. $\aleph_{m}$ is constant for $\left|p_{3}\right| \leq \sqrt{|q E|}(\sqrt{|q E|} T / 2-K)$ and for $T>>T_{0}$, and decreases rapidly for $\left|p_{3}\right|>\sqrt{|q E|}(\sqrt{|q E| T} / 2+K)$. The contribution to the integral (80) from the intermediate region can be estimated as $2 \sqrt{|q E|} K$. This implies

$$
n_{p_{\perp}}=\frac{J \sqrt{|q E|}}{(2 \pi)^{3}}\left[\sqrt{|q E|} T e^{-\pi \lambda}+O(K)\right]
$$

where $J$ is the number of the spin degrees of freedom ( $J=1$ for scalar particles and $J=2$ for fermions). Thus, the $p_{\perp}$ distribution density of the particle production rate has the form

$$
\frac{d n_{p_{\perp}}}{d T}=\frac{J|q E|}{(2 \pi)^{3}} e^{-\pi \lambda} .
$$

The total number of particles created per unit volume is given by

$$
\frac{\aleph}{V}=J \frac{(q E)^{2} T}{(2 \pi)^{3}} \exp \left\{-\pi \frac{M^{2}}{|q E|}\right\} .
$$


Suppose that, in addition to the external electric field, there exists a parallel constant magnetic field $B$. For definiteness, let us choose its potentials as $A_{\mu}^{B}=B x^{2} \delta_{\mu}^{1}$. Then, the complete set of quantum numbers that describes particles in such a background is $\left(p_{1}, n_{B}, p_{3}, r\right), n_{B}=0,1, \ldots$, and $\lambda=\left(M^{2}+|q B|\left(2 n_{B}+1-r\right)\right)|q E|^{-1}$. Substituting this $\lambda$ into eqs. (77) and (81), we obtain the differential mean numbers and $p_{\perp}$-distribution density of particles created per unit volume, respectively. One can see that the presence of the magnetic field essentially changes the energy spectrum of the transversal modes (which used to be $\mathbf{p}_{\perp}^{2}$ in the absence of the magnetic field) and also the form of $\lambda$, but does not change the dependence of $\aleph_{m}$ and $n_{p_{\perp}}$ on $\lambda$. This is related to the fact that the magnetic field itself does not produce work acting on charged particles, and, therefore, does not create particles. Then, the total number of particles created per unit volume is given by

$$
\frac{\aleph}{V}=\frac{J q^{2} E B T[\cosh (\pi B / E)]^{(1+\kappa) / 2}}{8 \pi^{2} \sinh (\pi B / E)} \exp \left\{-\pi \frac{M^{2}}{|q E|}\right\},
$$

where $\kappa=+1$ for fermions and $\kappa=-1$ for scalar particles.

For a strong electric field, $M^{2} /|q E| \lesssim 1,(B=0)$, and large $T$, the energy density of created pairs reads $\mathcal{E}=|q E| T \aleph / V$; see 42 . We can neglect the back-reaction of particles created by the electric field in case their energy density is essentially smaller than the energy density of the electric field, $\mathcal{E} \ll E^{2} / 8 \pi$. Consequently, the concept of a strong constant electric field is consistent only if the following condition holds true:

$$
1 \ll|q E| T^{2} \ll \frac{\pi^{2}}{J q^{2}} \exp \left\{\pi \frac{M^{2}}{|q E|}\right\} .
$$

Following [4, we represent the asymptotic formula (77) in a universal form:

$$
\aleph_{m}=\exp \left\{-2 \pi \frac{\omega_{m}}{g}\right\},
$$

where $\omega_{m}$ is the work of an external field creating a particle from a pair in a given state $m$,

$$
\omega_{m}=\frac{1}{2}\left[p_{0}\left(t_{f}\right)+p_{0}\left(t_{i}\right)+\Delta \epsilon_{v a c}\right]
$$

where $p_{0}\left(t_{f}\right)$ and $p_{0}\left(t_{i}\right)$ are particle energies at the final time instant $t_{f}$ and at an initial time instant $t_{i}$, respectively, and $\Delta \epsilon_{v a c}$ is a shift of the vacuum energy due to the time evolution. The quantity $g$ is the classical acceleration of a particle at the final time instant. In the case of a $T$-constant field, one can obtain

$$
\omega_{m}=\frac{M^{2}+\mathbf{p}_{\perp}^{2}}{2 p_{0}\left(t_{f}\right)}=\frac{\lambda}{T}, g=\frac{|q E|}{p_{0}\left(t_{f}\right)}=\frac{2}{T} .
$$

Thus, we can see that the differential mean values (86) are given, in fact, by the Boltzmann formula with the temperature $\theta=\frac{g}{2 \pi k_{B}}$ (where $k_{B}$ is the Boltzmann constant), the latter having literally the Hawking form $[8]$; see below.

We recall that the Hawking result for bosons created by the static gravitational field of a black hole in a specific thermal environment has the Planck form

$$
\aleph_{m}=\left[\exp \left\{2 \pi \frac{\omega_{m}}{g_{(H)}}\right\}-1\right]^{-1}
$$

Here, $\omega_{m}$ is the energy of a created particle and the Hawking temperature reads $\theta_{(H)}=\frac{g_{(H)}}{2 \pi k_{B}}$, where $g_{(H)}=\frac{G M}{r_{g}^{2}}$ is the free-fall acceleration at the gravitational radius $r_{g}$ of a black hole with mass $M$. In this case of a quasi-static gravitation field, the evolution shift of the vacuum energy is $\Delta \epsilon_{\text {vac }}=0$, so that one identifies the work $\omega_{m}$ (we have introduced) with the energy of a particle in formula (87). It is also known [43] that an observer which moves with a constant acceleration $g_{(R)}$ (with respect to its proper time) will probably register in the Minkowski vacuum some particles (Rindler particles). The distribution of Rindler bosons has the same Planck form (87), where one needws to replace $g_{(H)}$ by $g_{(R)}$, so that the corresponding temperature is $\theta_{(R)}=\frac{g_{(R)}}{2 \pi k_{B}}$.

It is a direct consequence of the equivalence principle that the effective temperature $\theta$ of distribution (86) has literally the Hawking form. The different form of distributions can be caused by essentially 
different structures of the Fock space in both cases. We believe that the Planck distribution arises necessarily due to the appearance of an event horizon (there is a boundary of the domain of the Hamiltonian), that is, due to the condition for which the space domains of the particle and antiparticle vacua are not the same. On the other hand, the final state can be treated as an equilibrium state. In contrast to this, in a uniform electric field we deal, in fact, with both the particle vacuum and the antiparticle vacuum defined over the entire space, that is, these space domains coincide. In this case, the mixed state of particles (antiparticles) described by the $\check{\rho}_{v+}\left(\check{\rho}_{v-}\right)$ density matrix of Sec. 3 can be represented as a pure state in an extended phase space where the space domains of both the particle vacuum and the antiparticle vacuum are the same, being a state of a far-from-equilibrium system. Let us note that in the framework of a semi-classical description at $\omega_{m} / g<<1$ the Boltzmann spectrum closely approaches the Planck spectrum.

\subsection{Soft parton production by $\mathrm{SU}(3)$ chromoelectric field}

As mentioned in Introduction, in QCD there exist physical situations that are quite efficiently described by the chromoelectric flux tube model. In this model, the back-reaction of created pairs induces a gluon mean field and plasma oscillations (see [4] and references therein). It appears that the calculation of particle-creation in this model requires the application of the general formalism of QFT for pairproduction at a finite temperature and at zero temperature both from vacuum and from many-particle states (see [26, 36, 45] for physical reasons). The study of various time scales in heavy-ion collisions shows that the stabilization time $T_{0}$ is far smaller than the period of plasma and mean-field oscillations. Then, according to condition (85), the approximation of a strong $T$-constant chromoelectric field can be used in treating such collisions during a period when the produced partons can be considered as weakly coupled due to the property of asymptotic freedom in QCD. It may also be reasonable to neglect dynamical backreaction effects and to consider only pair-production from vacuum by a constant $S U(3)$ chromoelectric field.

Here, we would like to turn our attention to results obtained in QCD with a constant $S U(3)$ chromoelectric field $E^{a}(a=1, \ldots, 8)$ along the $x^{3}$-axis; see [12. In this work, the imaginary parts of one-loop effective actions for quarks $S^{\text {quark }}$ and gluons $S^{\text {gluon }}$ have been calculated via gauge-invariant $p_{\perp}$ distributions $S_{p_{\perp}}^{q u a r k}$ and $S_{p_{\perp}}^{\text {gluon }}$ respectively. They have the form

$$
\begin{aligned}
& \operatorname{Im} S^{\text {quark }}=\int d^{2} p_{\perp} \operatorname{Im} S_{p_{\perp}}^{\text {quark }}, \operatorname{Im} S^{\text {gluon }}=\int d^{2} p_{\perp} \operatorname{Im} S_{p_{\perp}}^{\text {gluon }}, \\
& \operatorname{Im} S_{p_{\perp}}^{\text {quark }}=-\frac{V T}{8 \pi^{3}} \sum_{j=1}^{3}\left|q E_{(j)}\right| \ln \left(1-e^{-\pi \lambda_{(j)}}\right), \\
& \operatorname{Im} S_{p_{\perp}}^{\text {gluon }}=\frac{V T}{8 \pi^{3}} \sum_{j=1}^{3}\left|q \tilde{E}_{(j)}\right| \ln \left(1+e^{-\pi \tilde{\lambda}_{(j)}}\right), \\
& \lambda_{(j)}=\frac{M^{2}+\mathbf{p}_{\perp}^{2}}{\left|q E_{(j)}\right|}, \quad \tilde{\lambda}_{(j)}=\frac{\mathbf{p}_{\perp}^{2}}{\left|q \tilde{E}_{(j)}\right|},
\end{aligned}
$$

where $E_{(j)}$ are the eigenvalues of the matrix $i T^{a} E^{a}$ for the fundamental representation of $S U(3) ; \tilde{E}_{(j)}$ are the positive eigenvalues of the matrix $i f^{a b c} E^{c}$ for the adjoint representation of $S U(3)$; and $q$ is the coupling constant. These eigenvalues are the following gauge-invariant quantities:

$$
\begin{aligned}
& E_{(1)}=\sqrt{C_{1} / 3} \cos \theta, E_{(2)}=\sqrt{C_{1} / 3} \cos (2 \pi / 3-\theta), \\
& E_{(3)}=\sqrt{C_{1} / 3} \cos (2 \pi / 3+\theta),
\end{aligned}
$$

where $\theta$ is given by $\cos ^{2} 3 \theta=3 C_{2} / C_{1}^{3}$, and

$$
\begin{aligned}
& \tilde{E}_{(1)}=\left[\frac{C_{1}}{2}(1-\cos \tilde{\theta})\right]^{1 / 2}, \tilde{E}_{(2)}=\left[\frac{C_{1}}{2}\left(1+\cos \left(\frac{\pi}{3}-\tilde{\theta}\right)\right)\right]^{1 / 2}, \\
& \tilde{E}_{(3)}=\left[\frac{C_{1}}{2}\left(1+\cos \left(\frac{\pi}{3}+\tilde{\theta}\right)\right)\right]^{1 / 2},
\end{aligned}
$$


where $\tilde{\theta}$ is given by $\cos ^{3} \tilde{\theta}=-1+6 C_{2} / C_{1}^{3}$. Here, $C_{1}$ and $C_{2}$ are Casimir invariants for $S U(3)$,

$$
C_{1}=E^{a} E^{a}, \quad C_{2}=\left(d_{a b c} E^{a} E^{b} E^{c}\right)^{2},
$$

where $d_{a b c}$ is a symmetric invariant tensor in the adjoint representation of $S U(3)$. Then, the probabilities $P^{v}$ for a vacuum to remain a vacuum are found, for both quarks and gluons, from relation (60). However, formulas for parton production rates obtained in [12] hold only in the approximation $2 \operatorname{Im} S_{p_{\perp}}^{\text {quark }} \ll 1$ and $2 \operatorname{Im} S_{p_{\perp}}^{\text {gluon }} \ll 1$ by virtue of the arguments that we present at the end of Sbsection 3.2. To obtain exact results, we can use the following line of reasoning. The results (88) can be treated as those obtained in the case of a $T$-constant chromoelectric field when the integration over the longitudinal momentum and the summation over the spin and color degrees of freedom have been carried out. Then, using relation (69), we can extract from representation (88) an exact expression for $p_{\perp}$ distribution densities of quarks $n_{p_{\perp}}^{\text {quark }}$ and gluons $n_{p_{\perp}}^{\text {gluon }}$ produced per unit volume. Those are

$$
n_{p_{\perp}}^{q u a r k}=\frac{T}{4 \pi^{3}} \sum_{j=1}^{3}\left|q E_{(j)}\right| e^{-\pi \lambda_{(j)}}, n_{p_{\perp}}^{\text {gluon }}=\frac{T}{4 \pi^{3}} \sum_{j=1}^{3}\left|q \tilde{E}_{(j)}\right| e^{-\pi \tilde{\lambda}_{(j)}},
$$

where $T$ is a sufficiently large action period of a constant field. The $p_{\perp}$ distribution densities of particle production rates can be found as $d n_{p_{\perp}}^{\text {quark }} / d T$ and $d n_{p_{\perp}}^{\text {gluon }} / d T$, respectively. The total numbers of quarks and gluons created per unit volume can be obtained from (89) as follows:

$$
\frac{\aleph^{\text {quark }}}{V}=\frac{T}{4 \pi^{3}} \sum_{j=1}^{3}\left(q E_{(j)}\right)^{2} \exp \left\{-\pi \frac{M^{2}}{\left|q E_{(j)}\right|}\right\}, \frac{\aleph^{\text {gluon }}}{V}=\frac{3 T q^{2} C_{1}}{8 \pi^{3}},
$$

where the relation $\sum_{j=1}^{3} \tilde{E}_{(j)}^{2}=3 C_{1} / 2$ is used. Taking into account the relation $\sum_{j=1}^{3} E_{(j)}^{2}=C_{1} / 2$, one can see from (90) that in a sufficiently strong field $E_{(j)}, M^{2} /\left|q E_{(j)}\right| \ll 1$, the densities of created quarks and gluons are related by $\aleph^{q u a r k} / V=\aleph^{\text {gluon }} / 3 \mathrm{~V}$.

We can see that the $(j)$ - terms in expressions (88) and (90) can be interpreted as those which are obtained for Abelian-like electric fields $E_{(j)}$ and $\tilde{E}_{(j)}$, respectively. The maxima of the fields are restricted by the conditions $\left|E_{(j)}\right| \leq \sqrt{C_{1} / 3}$ and $\left|\tilde{E}_{(j)}\right| \leq \sqrt{C_{1}}$. Therefore, in order to study the validity of the constant $S U(3)$ chromoelectric field approximation we need to take into account only the energy density of gluons created by the field $\tilde{E}_{(j)}$. We know from the previous subsection that this energy per a single pair is $\left|q \tilde{E}_{(j)}\right| T$. Then, the total energy density of created gluons reads

$$
\mathcal{E}=\frac{T^{2}}{4 \pi^{3}} \sum_{j=1}^{3}\left|q \tilde{E}_{(j)}\right|^{3} \lesssim|q| \sqrt{C_{1}} T \frac{\aleph^{\text {gluon }}}{V} .
$$

One can neglect the back-reaction of these gluons created by the chromoelectric field only if $\mathcal{E} \ll C_{1} / 8 \pi$. Finally, the condition of validity of the $T$-constant $S U(3)$ chromoelectric field approximation can be written as

$$
1 \ll|q| \sqrt{C_{1}} T^{2} \ll \frac{\pi^{2}}{3 q^{2}} .
$$

Therefore, we can see that the $T$-constant $S U(3)$ chromoelectric field approximation is consistent during the period when the produced partons can be treated as weakly coupled.

Recently, it has been discovered (see, [46]), in the framework of the Color Glass Condensate Approach to the description of heavy ion collisions, that shortly after the collision the system contains both longitudinal chromoelectric and chromomagnetic fields. In such a chromomagnetic field, the above-described soft parton production is changed. First of all, the energy spectrum of the transversal modes becomes different, which implies that the expressions for $\mathbf{p}_{\perp}^{2}$ that enter $\lambda_{(j)}$ and $\tilde{\lambda}_{(j)}$ are different from the case of pure chromoelectric field. For example, if the chromomagnetic field $B^{c}$ is uniform then $\mathbf{p}_{\perp}^{2}=\left|q B^{c}\right|\left(2 n_{B}+1-r\right)$, $n_{B}=0,1, \ldots$ This expression is similar to the case of QED, see Section 4.1. Here, the spin quantum number $r$ takes the values \pm 1 for quarks and $-2,0,+2$ for gluons. As in QED, the constant chromomagnetic field does not produce work acting on charged particles, and, therefore, does not create particles. The distribution densities of created quarks $n_{p_{\perp}}^{q u a r k}$ and gluons $n_{p_{\perp}}^{\text {gluon }}$ as functions of $\lambda_{(j)}$ and $\tilde{\lambda}_{(j)}$ do not change and are given by equations (89). 
Nevertheless, the above expression for $\mathbf{p}_{\perp}^{2}$ in a uniform chromomagnetic field is negative for $n_{B}=0$, $r=+2$. This is why such a configuration of the chromomagnetic field is unstable under quantum fluctuations. For the first time, it was mentioned in [47. Then, the problem has been discussed in numerous articles: for a review, see, e.g., [48, 49. One of the possible stable configurations of the longitudinal chromomagnetic field is the so-called flux-tube state, alias the spaghetti state (see [48 and references therein). An explicit form of the spectra $\mathbf{p}_{\perp}^{2}$ in this field is unknown. Nevertheless, a stable chromomagnetic field is constant and longitudinal. This is sufficient for us to make the above conclusion that the dependence on $\lambda_{(j)}$ and $\tilde{\lambda}_{(j)}$, given by eq. (89), in the presence of a longitudinal chromomagnetic field remains unchanged.

In the following sections, we turn once again to particle creation by electric field in QED. The above discussion shows that it can be useful for understanding the effects of quark and gluon creation in QCD.

\subsection{Thermal-like distributions}

As has been mentioned, the thermalization stage of multiparticle production in ion-ion collisions at high energies is very important. On the other hand, as we know from Section 3, it is sometimes difficult to distinguish a real thermal equilibrium from a state where we have a one-particle thermal distribution. In this connection, we shall consider some simple examples when pair-creation by electric field can mimic a one-particle thermal distribution.

We recall that due to the screen of created pairs, the original electric field may have an exponential fall-off:

$$
E\left(x^{0}\right)=E e^{-x^{0} / \alpha} .
$$

The differential mean number of particles created from vacuum by this field has been calculated in [50]. The result is

$$
\aleph_{m}= \begin{cases}{\left[\frac{\cosh \left[\pi \alpha\left(\varepsilon+p_{3}^{\prime}\right)\right.}{\cosh \left[\pi \alpha\left(\varepsilon-p_{3}^{\prime}\right)\right]} e^{2 \pi \alpha \varepsilon}-1\right]^{-1} \text { Bose case, }} \\ {\left[\frac{\sinh \left[\pi \alpha\left(\varepsilon+p_{3}^{\prime}\right)\right]}{\sinh \left[\pi \alpha\left(\varepsilon-p_{3}^{\prime}\right)\right]} e^{2 \pi \alpha \varepsilon}+1\right]^{-1} \text { Fermi case }}\end{cases}
$$

where $\varepsilon=\sqrt{M^{2}+\mathbf{p}^{2}}$ and $p_{3}^{\prime}=p_{3} \operatorname{sgn}(q E)$. For $\pi \alpha\left|p_{3}\right| \ll 1$, these expressions coincide with the Bose and Fermi distributions at the temperature $\theta=\left(2 \pi k_{B} \alpha\right)^{-1}$, respectively.

Another example is the pulse of electric field (78). The differential mean number of particles created from vacuum by the sharp field pulse (78) at $\alpha|q E| / \varepsilon \ll 1$ can be extracted from the result [41] and has the form

$$
\aleph_{m}=\left\{\begin{array}{l}
\left(\pi q E \alpha^{2}\right)^{2}\left[\left(q E \alpha^{2}\right)^{2}+\left(p_{3} / \varepsilon\right)^{2}\right] \sinh ^{-2}(\pi \alpha \varepsilon) \text { Bose case } \\
\left(\pi q E \alpha^{2}\right)^{2}\left[1-\left(p_{3} / \varepsilon\right)^{2}\right] \sinh ^{-2}(\pi \alpha \varepsilon) \text { Fermi case }
\end{array}\right.
$$

When the ratio $\left|p_{3}\right| / \varepsilon$ is sufficiently small and the external field is not strong, $\varepsilon / \sqrt{|q E|} \gg 1$, there is a range of values $\alpha, \pi \alpha \epsilon \gg 1$, in which distributions (94) have the Boltzmann form with the temperature $\theta=\left(2 \pi k_{B} \alpha\right)^{-1}$.

As we note in the previous subsection, depending on the details of the chromoelectric flux tube model, its stage and field strength, pair production both from vacuum and from many-particle states by a $T$ constant electric field may be relevant. The well-known asymptotic form (77) of the differential mean numbers for pairs created from vacuum by a constant electric field can lead to a thermal-like distribution of created pairs if, for example, the chromoelectric string tension undergoes Gaussian fluctuations 51. This implies a modification of the original flux tube model by introducing a fluctuating string tension. In case the original flux tube model still holds, nevertheless, the differential mean numbers of pairs created by a $T$-constant electric field can be represented as the Boltzmann distribution (86) with the temperature $\theta=\left(\pi k_{B} T\right)^{-1}$, as has been seen in Subsection 4.1. Then, it may be reasonable to examine the phenomenological model with a slowly oscillated mean electric field and suppose that the pair creation by the mean field during the semiperiod of oscillation can be effectively approximated by a $T$-constant electric field (we recall that the time scale of stabilization $T_{0}$ is far smaller than the period of oscillations). In this case, the electric field produces pairs for a semi-period of oscillation in the presence of pairs created at previous stages. This is the way to take into account the effects of back-reaction in such a model. In other words, we are going to consider pair-creation from the initial state given by a distribution of previously created particles. Formula (59) is relevant in this analysis. 
Starting from the initial vacuum state, one has $\Delta N_{m}=\aleph_{m}$, where $\aleph_{m}$ belongs to the asymptotic form (77). Then, at the end of the first stage, when the mean field is depleted for the first time, the distribution of particles (being equal to that of antiparticles) is $N_{m}^{(1)}=\aleph_{m}$. During the second stage, the direction of the mean field is opposite to the field direction at the first stage. Due to the condition of stabilization, this is of no importance, since $\aleph_{m}$ is an even function of $q E$. Thus, when the mean field is depleted for the second time, from (59) there follows the equality

$$
N_{m}^{(2)}=\aleph_{m}+\left(1-2 \kappa \aleph_{m}\right) N_{m}^{(1)},
$$

and at the end of the $n$-th stage,

$$
N_{m}^{(n)}=\aleph_{m}+\left(1-2 \kappa \aleph_{m}\right) N_{m}^{(n-1)} .
$$

Consequently, the total number of particles created at the end of the $n$-th stage is

$$
N_{m}^{(n)}=\aleph_{m} \sum_{l=0}^{n-1}\left(1-2 \kappa \aleph_{m}\right)^{l} .
$$

We have this result if the created particles do not leave the region of the active field. In order to take into account the possible loss of particles due to interaction, movement, etc., we also assume that the total number of particles at the initial state of the $n$-th stage is less than the number $N^{(n-1)}$ of particles created at the end of the $(n-1)$-th stage and is $\gamma N^{(n-1)}$, where $\gamma<1$ is the factor of loss. Then, the modified relation is

$$
N_{m}^{(n)}=\aleph_{m}+\left(1-2 \kappa \aleph_{m}\right) \gamma N_{m}^{(n-1)},
$$

and we finally have

$$
N_{m}^{(n)}=\aleph_{m} \sum_{l=0}^{n-1} \gamma^{l}\left(1-2 \kappa \aleph_{m}\right)^{l} .
$$

Supposing that $\gamma$ is constant, one can calculate the sum in (96),

$$
N_{m}^{(n)}=\aleph_{m} \frac{1-r^{n}}{1-r}, r=\gamma\left(1-2 \kappa \aleph_{m}\right) .
$$

For fermions, $\kappa=+1$, then $N_{m}^{(n)} \leq 1$. Energy dissipation after a period of oscillation is estimated (for real parameters of heavy-ion collisions) not to be large, so that damping is small and the number of oscillations can be quite large; damping decreases with an increasing field strength. If the number of cycles is sufficiently large, we get the limiting thermal-like distribution

$$
N_{m}^{\Sigma}=\frac{\aleph_{m}}{1-\gamma\left(1-2 \kappa \aleph_{m}\right)}=\frac{1}{2 \gamma} \cdot \frac{1}{e^{\pi \lambda}(1-\gamma) / 2 \gamma+\kappa} .
$$

In other words, the system reaches a quasi-equilibrium state. For bosons, $\kappa=-1$, then $N_{m}^{(n)}$ increases. This is the phenomenon of resonance, and the increase can be either limited or unlimited depending on the factor $\gamma$. The increase is limited as long as $r<1$. In this case, formula (98) is valid for bosons as well. We can see that back-reaction-induced plasma oscillations can reach a quasi-stationary form specified by thermal-like distribution for both bosons and fermions.

\subsection{Particle creation at finite temperature}

We are now ready to present explicitly the mean number of (anti)particles in the mode $m$ (with finite longitudinal momenta, $\left|p_{3}\right| \leq \sqrt{|q E|}(\sqrt{|q E|} T / 2-K)$ ) for the final state of evolution in a quasi-constant field from the initial thermodynamical equilibrium, $N_{m}^{(\zeta)}(i n)=\left(e^{E_{m}}+\kappa\right)^{-1}$, at equal chemical potentials $\mu^{(+)}=\mu^{(-)}=\mu,(\mu<M$ for bosons $)$,

$$
N_{m}^{(\zeta)}=\left(e^{E_{m}}+\kappa\right)^{-1}+e^{-\pi \lambda}\left(\tanh \left(E_{m} / 2\right)\right)^{\kappa},
$$

where $E_{m}=\beta\left(\varepsilon_{m}-\mu\right), \varepsilon_{m}=\sqrt{M^{2}+\mathbf{p}_{\perp}^{2}+\left(\pi_{3}\right)^{2}}, \pi_{3}=p_{3}+q E T / 2$, and it is implied that $\lambda$ is given by (76). This result for the electric field coincides with the one obtained in [24]. Due to the effect of 
stabilization, it seems that the time-dependence of the final distributions in question is absent. However, the integral mean numbers vary as long as a quasi-constant field is active.

It is of interest to establish a general behavior of the integral mean numbers of created particles when the effects of switching on and off are negligible. As has been shown above, we can satisfy this condition by selecting the action time $T$ of the $T$-constant field $\left(T>>T_{0}\right)$ as an effective period of pair creation. It is implied that, in general, the final time instant, $t_{f}$, and the initial time instant, $t_{i}$, are so selected that the quasi-constant field is closely approximated by the $T$-constant field for a period from $t_{i}$ to $t_{f}$, and $t_{f}-t_{i}=T$.

Let us estimate the sum over the longitudinal momentum $p_{3}$ of $\Delta N_{m}$ in (59), which is the mean number of particles created with all the possible values $p_{3}$. As above, $\sum_{\mathbf{p}} \rightarrow \frac{V}{(2 \pi)^{3}} \int d \mathbf{p}$, and the distribution $\aleph_{m}$ plays the role of the cut-off factor for the integral over $p_{3}$. Then, one can conclude that the $p_{\perp}, r$ distribution density of particles produced per unit volume is finite and can be presented as follows:

$$
\begin{aligned}
& n_{\mathbf{p}_{\perp}, r}^{c r}=\frac{1}{(2 \pi)^{3}} \int_{-\infty}^{\infty} \Delta N_{m} d p_{3} \\
& =\frac{1}{(2 \pi)^{3}}\left[e^{-\pi \lambda} \int_{-|q E| T / 2}^{|q E| T / 2} n_{m}(\beta) d p_{3}+\sqrt{|q E|} O(K)\right], \\
& n_{m}(\beta)=\left(\tanh \left(E_{m} / 2\right)\right)^{\kappa} .
\end{aligned}
$$

From (100), one can estimate the $p_{\perp}, r$ distribution density of the particle production rate,

$$
\frac{d n_{\mathbf{p}_{\perp}, r}^{c r}}{d T}=\left.\frac{|q E|}{(2 \pi)^{3}} n_{m}(\beta)\right|_{\pi_{3}=|q E| T} e^{-\pi \lambda} .
$$

We have $(q E T)^{2} \gg M^{2}+\mathbf{p}_{\perp}^{2}$, according to the condition of stabilization. Then, the high and low temperature limits for the production rate are only defined by the final longitudinal kinetic momentum $|q E| T$ and the temperature $\Theta$ relation, $\beta|q E| T \ll 1$ and $\beta|q E| T \gg 1$, respectively. For simplicity, we assume that $|q E| T \gg \mu$. Considering these limits, one obtains for the temperature-dependent term in (101)

$$
\begin{aligned}
& \left.n_{m}(\beta)\right|_{\pi_{3}=|q E| T}=1-2 \kappa e^{-\beta|q E| T}, \beta|q E| T \gg 1, \\
& \left.n_{m}(\beta)\right|_{\pi_{3}=|q E| T}=[\beta|q E| T / 2]^{\kappa}, \beta|q E| T \ll 1 .
\end{aligned}
$$

We can see that at high temperatures the rate $\frac{d n_{\mathbf{P}, r}^{c r}}{d T}$ is time-dependent; it is much lower than the zerotemperature value but increases for fermions, and is considerably higher than the zero temperature value but decreases for bosons. Consequently, the frequently used notion of a number of particles created per unit of time makes sense only at low temperatures and in this limit it coincides with the zero-temperature value of the production rate. We consider two temperature limits for the $p_{\perp}, r$ distribution density (100): low temperatures at $\beta\left(\varepsilon_{\perp}-\mu\right) \gg 1, \varepsilon_{\perp}=\sqrt{M^{2}+\mathbf{p}_{\perp}^{2}}$, when all the energies of the particles created in the modes with a given $\mathbf{p}_{\perp}$ are considerably higher than the temperature $\Theta$, and high temperatures, at $\beta|q E| T \ll 1$, when all the energies of the created particles are much lower than the temperature $\Theta$,

$$
\begin{aligned}
& n_{\mathbf{p}_{\perp}, r}^{c r}=\frac{\sqrt{|q E|}}{(2 \pi)^{3}}\left[\sqrt{|q E| T} e^{-\pi \lambda}+O(K)\right], \kappa= \pm 1, \quad \beta\left(\varepsilon_{\perp}-\mu\right) \gg 1, \\
& n_{\mathbf{p}_{\perp}, r}^{c r}=\frac{\beta|q E|}{(2 \pi)^{3}}\left[|q E| T^{2} / 2+O(\sqrt{|q E| T})\right] e^{-\pi \lambda}, \kappa=+1, \beta|q E| T \ll 1, \\
& n_{\mathbf{p}_{\perp}, r}^{c r}=\frac{\sqrt{|q E|}}{(2 \pi)^{3}}\left[\frac{4}{\beta \sqrt{|q E|}} \ln (\sqrt{|q E|} T / K) e^{-\pi \lambda}+O(K)\right], \quad \kappa=-1, \beta|q E| T \ll 1 .
\end{aligned}
$$

The result at low temperatures is not different from the zero-temperature result 4 within the accuracy of our analysis. Integrating expressions (102) over $\mathbf{p}_{\perp}$, one finds the total number of particles created per 
unit volume at the low-temperature and high-temperature limits, respectively:

$$
\begin{aligned}
& \frac{N^{c r}}{V}=J \frac{(q E)^{2} T}{(2 \pi)^{3}} e^{-\pi M^{2} /|q E|}, \quad \beta(M-\mu) \gg 1, \\
& \frac{N^{c r}}{V}=\frac{\beta|q E|^{3} T^{2}}{(2 \pi)^{3}} e^{-\pi M^{2} /|q E|}, \kappa=+1, \beta|q E| T \ll 1, \\
& \frac{N^{c r}}{V}=\frac{|q E| \ln (\sqrt{|q E| T})}{2 \pi^{3} \beta} e^{-\pi M^{2} /|q E|}, \quad \kappa=-1, \beta|q E| T \ll 1,
\end{aligned}
$$

where the summation over $r= \pm 1$ is carried out for the fermions, and only the leading $T$-dependent terms are presented. From (102), (103), we can see that the values of the integral mean numbers for fermions at high temperatures are much lower than the corresponding values at low temperatures. For bosons, the integral mean numbers at high temperatures are considerably higher than the corresponding values at low temperatures.

As mentioned in Introduction, thermally-influenced pair production by a constant electric field has been investigated in several approaches [26, 24, 25, 27]. The results are quite contradictory, varying from the absence of creation to the rates of fermion production higher than the rate at zero temperature. Now, we are ready to discuss these contradictions. As has been shown above, the initial thermal distribution affects the number of states in which pairs are created by a quasi-constant field. Hence, pair-production exists at any temperatures, and, in particular, the fermion production rate cannot be higher than the rate at zero temperature, by any means. Note that our calculations are based on the generalized Furry representation developed especially for the case of vacuum instability in accordance with the basic principles of quantum field theory. On the other hand, all the conclusions of 26, 27] concerning the pair production rate and/or the mean numbers of pairs created at non-zero temperatures are based on either the standard real-time, or imaginary-time, one-loop effective actions. However, such formalisms do not work in the presence of unstable modes. The real part of the standard effective action describes the effects of vacuum polarization and has nothing to do with the time-dependent conduction current of created pairs. For example, this can be observed at zero temperature (see [42]). In this case, the information concerning pair-creation comes from the imaginary part of the standard effective action. The extension of real-time techniques for finite-temperature quantum electrodynamics with unstable vacuum has been presented in 32. In this article, one can see that the relevant Green functions in a constant electric field are quite different from the standard proper-time representation given by Schwinger. Then, the relevant real-time one-loop effective action must be different from the standard on 5 . The standard imaginary-time formalism, obtained under the assumption of a thermal equilibrium and the appearance of a contradiction with the Pauli exclusion principle, shows that the attempts of generalization to farfrom-equilibrium systems have failed. The functional Schrödinger picture used in 25] to calculate the $N^{c r}$ at high temperatures seems relevant; its asymptotic expressions for $N^{c r}$ at high temperatures are in agreement with our expressions in (103).

Acknowledgement We thank Yuri Sinyukov for useful discussions, and, in particular, for calling our attention to Ref. [14.

S.P.G. and J.L.T. thank FAPESP for support. D.M.G. acknowledges the support of FAPESP and CNPq. S.P.G. is grateful to Universidade Estadual Paulista (Campus de Guaratinguetá) and Universidade de São Paulo for hospitality.

\section{Appendix}

I. For both the Bose and Fermi cases, the following relations hold:

$$
\begin{aligned}
& a e^{a^{\dagger} D a}=e^{a^{\dagger} D a} e^{D} a, \quad a^{\dagger} e^{a^{\dagger} D a}=e^{a^{\dagger} D a} a^{\dagger} e^{-D}, \\
& e^{a^{\dagger} D a}=: \exp \left\{a^{\dagger}\left(e^{D}-1\right) a\right\}:,
\end{aligned}
$$

\footnotetext{
${ }^{5}$ We will present the relevant real-time one-loop effective action elsewhere.
} 
where $D$ is a matrix. To prove (105), let us consider the operator function $F(s)=e^{s a^{\dagger} D a}$, where $s$ is a parameter. The function is a solution of the following equation:

$$
\frac{d F(s)}{d s}=a^{\dagger} \operatorname{DaF}(s), F(0)=1 .
$$

Using relation (104), we can rewrite the right-hand side of the equation as follows:

$$
\frac{d F(s)}{d s}=a^{\dagger} F(s) D e^{s D} a, \quad F(0)=1
$$

Now, we can verify that a solution of such an equation reads

$$
F(s)=: \exp \left\{a^{\dagger}\left(e^{s D}-1\right) a\right\}: .
$$

Setting $s=1$, we justify (105).

II. We often use the well-known relation

$$
e^{\lambda a} e^{a^{\dagger} \tilde{\lambda}}=e^{a^{\dagger} \tilde{\lambda}} e^{\lambda a} e^{\lambda \tilde{\lambda}}
$$

where $\lambda$ and $\tilde{\lambda}$ are Grassmann-odd or Grassmann-even variables depending on statistics. For a product of two normal forms, there holds a generalization of (106), namely,

$$
: e^{a^{\dagger} D a}:: e^{a^{\dagger} \tilde{D} a}:=: e^{a^{\dagger}(D+\tilde{D}+D \tilde{D}) a}:
$$

where $D$ and $\tilde{D}$ are some matrices.

III. The projection operator on the vacuum state can be written as follows:

$$
P_{0}=|0\rangle\langle 0|=: e^{-a^{\dagger} a}:
$$

Such a representation was first used by Berezin [33]. One can see that the operator $P_{0}$ obeys the equations

$$
a P_{0}=0, P_{0} a^{\dagger}=0, P_{0}|0\rangle=|0\rangle .
$$

Using the Wick theorem, one can see that $: e^{-a^{\dagger} a}:$ is a solution of these equations.

IV. The trace of a normal product of creation and annihilation operators can be calculated by using the following path integral representation. Let $X\left(a^{\dagger}, a\right)$ be an operator expression of creation and annihilation operators, $a$ and $a^{\dagger}$. Then the trace of its normal form

$$
\operatorname{tr}\left\{: X\left(a^{\dagger}, a\right):\right\}=\sum_{M=0}^{\infty} \sum_{\{m\}}(M !)^{-1}\left\langle 0\left|a_{m_{M}} \ldots a_{m_{1}}: X\left(a^{\dagger}, a\right): a_{m_{1}}^{\dagger} \ldots a_{m_{M}}^{\dagger}\right| 0\right\rangle,
$$

can be expressed as the following vacuum mean value:

$$
\operatorname{tr}\left\{: X\left(a^{\dagger}, a\right):\right\}=\left\langle 0\left|T: X\left(a^{\dagger}, a\right): e^{a\left(t_{f}\right) a^{\dagger}\left(t_{i}\right)}\right| 0\right\rangle
$$

where the notation $a=a\left(t_{f}\right), a^{\dagger}=a^{\dagger}\left(t_{i}\right)$ is used for the operators $a$ to the left of : $X\left(a^{\dagger}, a\right):$ and $a^{\dagger}$ to the right of : $X\left(a^{\dagger}, a\right):$, whereas $T$ is the ordering operator putting $a\left(t_{f}\right)$ to the left of : $X\left(a^{\dagger}, a\right):$ and $a^{\dagger}\left(t_{i}\right)$ to the right of : $X\left(a^{\dagger}, a\right)$ :. Using either the Berezin path integral or the Gaussian integral over ordinary variables, depending on statistics, one can rewrite (109) as follows:

$$
\operatorname{tr}\left\{: X\left(a^{\dagger}, a\right):\right\}=\left\langle 0\left|\int \exp \left\{\kappa \lambda^{*} \lambda+\lambda^{*} a\right\}: X\left(a^{\dagger}, a\right): \exp \left\{a^{\dagger} \lambda\right\} \Pi d \lambda^{*} d \lambda\right| 0\right\rangle,
$$

where $a\left(t_{f}\right)=a$ and $a^{\dagger}\left(t_{i}\right)=a^{\dagger}$ are used after rewriting.

\section{References}

[1] O. Klein, Z. Phys. 53 (1929) 157; F. Sauter, Z. Phys. 69 (1931) 742; F. Sauter, Z. Phys. 73 (1931) 547. 
[2] J. Schwinger, Phys. Rev. 82 (1951) 664.

[3] A.I. Nikishov, Sov. Phys. JETP 30 (1970) 660; A.I. Nikishov, in Quantum Electrodynamics of Phenomena in Intense Fields, Proc. P.N. Lebedev Phys. Inst. 111 (Nauka, Moscow, 1979) 153; V.G. Bagrov, D.M. Gitman and Sh.M. Shvartsman, Sov. Phys.-JETP 41 (1975) 191; D.M. Gitman, V.M. Shachmatov and Sh.M. Shvartsman, Sov. Phys. J. 4 (1975) 23; V.G. Bagrov, D.M. Gitman and Sh.M. Shvartsman, Sov. J. Nucl. Phys. 23 (1976) 394.

[4] S.P. Gavrilov and D.M. Gitman, Phys. Rev. D 53 (1996) 7162 arXiv:hep-th/9603152.

[5] A. Ringwald, Phys. Lett. B 510 (2001) 107; arXiv:hep-ph/0103185 arXiv:hep-ph/0304139 R. Alkofer, M.B. Hecht, C.D. Roberts, S.M. Schmidt, and D.V. Vinnik., Phys. Rev. Lett. 87 (2001) 193902; V.S. Popov, JETP Lett. 74 (2001) 133; I.M. Dremin, JETP Lett. 76 (2001) 185.

[6] J.R.S. Nascimento, I. Cho, A. Vilenkin, Phys. Rev. D 60 (1999) 083505 arXiv:hep-th/9902135.

[7] W. Greiner, B. Müller, and J. Rafelski, Quantum Electrodynamics of Strong Fields (Springer-Verlag, Berlin, 1985); W. Greiner and J. Reinhardt, Quantum Electrodynamics (Springer-Verlag, Berlin, 1994).

[8] S.W. Hawking, Commun. Math. Phys. 43 (1975) 199; Phys. Rev. D 14 (1976) 2460.

[9] I.D. Novikov and V. P. Frolov, Physics of black holes (Kluwer Academic, Dordrecht, 1989); R.M. Wald, Quantum field theory in curved spacetime and black hole thermodynamics (The University of Chicago Press, Chicago, 1994).

[10] A.A. Grib, S.G. Mamaev, and V.M. Mostepanenko, Vacuum Quantum Effects in Strong Fields, (Atomizdat, Moscow, 1988; Friedmann Laboratory Publishing, St. Petersburg, 1994); N.D. Birrell and P.C.W. Davies, Quantum Fields in Curved Space (Cambridge University Press, Cambridge 1994); I.L. Buchbinder, S.D. Odintsov and I.L. Shapiro, Effective Action in Quantum Gravity (IOP Publishing, Bristol and Philadelphia 1992).

[11] N. Seiberg, L. Susskind and N. Toumbas, JHEP 0006 (2000) 021; R. Gopakumar, J. Maldacena, S. Minwalla and A. Strominger, JHEP 0006 (2000) 036; P. Mukhopadhyay and A. Sen, JHEP 0211 (2002) 047.

[12] G.C. Nayak, and P. van Nieuwenhuizen, Phys. Rev. D 71 (2005) 125001 arXiv:hep-ph/0504070; G.C. Nayak, Phys. Rev. D 72 (2005) 125010 arXiv:hep-ph/0510052; F. Cooper and G.C. Nayak, Phys. Rev. D 73 (2006) 065005 arXiv:hep-ph/0511053.

[13] F.Gelis, K. Kajantie, and T. Lappi, Phys. Rev. Lett. 96 (2006) 032304 arXiv:hep-ph/0508229.

[14] D. Kharzeev, E. Levin, and K. Tuchin, arXiv:hep-ph/0602063.

[15] A. Casher, H. Neuberger, and S. Nussinov, Phys. Rev. D 20 (1979) 179; E.G. Gurvich, Phys. Lett. B 87 (1979) 386.

[16] I.A. Batalin, S.G. Matinyan, and G.K. Savvidy, Sov. J. Nucl. Phys. 26 (1977) 214; J. Ambjorn and R. Hughes, Phys. Lett. B 113 (1982) 305; Ann. Phys. (N.Y.) 145 (1983) 340.

[17] A. Yildiz and P. H. Cox, Phys. Rev. D 21 (1980) 1095; M. Claudson, A. Yildiz and P. H. Cox, Phys. Rev. D 22 (1980) 2022.

[18] J. Manjavidze and A. Sissakian, Phys. Rept. 346 (2001) 1; D.D. Dietrich, G.C. Nayak, and W. Greiner, Phys. Rev. D 64 (2001) 074006; J.D. de Deus, E.G. Ferreiro, C. Pajares, and R. Ugoccioni, Phys. Lett. B 581 (2004) 156.

[19] L. McLerran, and M. Gyulassy, Nucl. Phys. A 750 (2005) 30.

[20] Y. Schutz, J. Phys. G 30 (2004) S903.

[21] L.V. Gribov, E.M. Levin, and M.G. Ryskin, Phys. Rep. 100 (1983) 1; L. McLerran, and R. Venugopalan, Phys. Rev. D 49 (1994) 2233, 3352; 50 (1994) 2225; 59 (1999) 094002; A. Ayala, J. Jalilian-Marian, L. McLerran, and R. Venugopalan, Phys. Rev. D 53 (1996) 458; A.H. Mueller, and J. Qiu, Nucl. Phys. B 268 (1986) 427. 
[22] L. McLerran, Lectures given at the 40'th Schladming Winter School: Dense Matter, March 3-10 2001, arXiv:hep-ph/0104285 E. Iancu, A. Leonidov, L. McLerran, Lectures given at Cargese, France, 6-19 Aug 2001, arXiv:hep-ph/0202270 E.Iancu, R. Venugopalan, Quark Gluon Plasma 3, Eds. R.C. Hwa and X.N. Wang, World Scientific arXiv:hep-ph/0303204; H. Weigert, Prog. Part. Nucl. Phys. 55 (2005) 461 arXiv:hep-ph/0501087.

[23] A. Kovner, L. McLerran,, and H. Weigert, Phys. Rev. D 52 (1995) 6231.

[24] I.L. Bukhbinder, D.M. Gitman, and V.P. Frolov, Izv. Vuzov Fizika 23, N.6 (1980) 77 [English. transl.: Sov. Phys. J. 23 (1980) 529].

[25] J. Hallin and P. Liljenberg, Phys. Rev. D 52 (1995) 1150.

[26] A.K. Ganguly, P.K. Kaw, and J.C. Parikh, Phys. Rev. C 51 (1995) 2091.

[27] P.H. Cox, W.S. Hellman, and A. Yildiz, Ann. Phys. (N.Y) 154 (1984) 211; M. Loewe and J.C. Rojas, Phys. Rev. D 46 (1992) 2689; P. Elmfors and B.-S. Skagerstam, Phys. Lett. B 348 (1995) 141; 376 (1996) 330(E); A.K. Ganguly, arXiv:hep-th/9804134; H. Gies, Phys. Rev. D 60 (1999) 105002.

[28] D.M. Gitman, J. Phys. A 10 (1977) 2007; E.S. Fradkin, D.M. Gitman and S.M. Shvartsman, Quantum Electrodynamics with Unstable Vacuum (Springer-Verlag, Berlin 1991)

[29] I.L. Buchbinder and D.M. Gitman, Izv. V. Fiz. (Sov. Phys. J.) 3 (1979) 90 ; ibid 4 (1979) 55; ibid 7 (1979) 16; I.L. Buchbinder, E.S. Fradkin and D.M. Gitman, Fortschr. Phys. 29 (1981) 187.

[30] S.P. Gavrilov and D.M. Gitman, Report MIT, Massachusetts, 1991, CTP\#1995 (unpublished); S.P. Gavrilov, Russian Phys. J. 35, No. 7 (1992) 652; ibid, 35, No. 10 (1992) 969; ibid, 36, No. 3 (1993) 269; S.P. Gavrilov and D.M. Gitman, ibid, 36, No. 5 (1993) 448; Izv. Vuzov. Fiz. No. 4 (1995) 83.

[31] J. Ambjorn, and R. J. Hughes, and N.K. Nielsen Ann. Phys. (N.Y.) 150 (1983) 92.

[32] S.P. Gavrilov, D.M. Gitman, and E.S. Fradkin, Yad. Fiz. 46 (1987) 172 [English. transl.: Sov. J. Nucl. Phys. (USA) 46 (1987) 107].

[33] F. A. Berezin, The method of second quantization (Nauka, Moscow, 1965) [English transl.: Academic Press, New York (1966)].

[34] D.M. Gitman and V.P. Frolov, Yad Fiz. 28 (1978) 552; V.P. Frolov and D.M. Gitman, J. Phys. A. 15 (1978) 1329.

[35] N. Glendenning and T. Matsui, Phys. Lett. B 141 (1984) 419; G. Gatoff, A.K. Kerman, and T. Matsui, Phys. Rev. D 36 (1987) 114; A. Bialas, W. Czy, A. Dyrek, and W. Florkowski, Nucl. Phys. B $296(1988) 611$.

[36] Y. Kluger, E. Mottola, and J.M. Eisenberg, Phys. Rev. D 58 (1998) 125015.

[37] G.V. Dunne, Heisenberg-Euler effective Lagrangians: Basics and extensions, in Ian Kogan Memorial Volume, From fields to strings: Circumnavigating theoretical physics, Eds. M Shifman, A. Vainshtein and J. Wheater, World Scientific arXiv:hep-th/0406216.

[38] C. Itzykson and J.-B. Zuber, Quantum Field Theory (Mc-Graw Hill, New York,1980).

[39] D. Kharzeev and K. Tuchin, Nucl. Phys. A 753 (2005) 316 arXiv:hep-ph/0602063.

[40] S.P. Gavrilov, D.M. Gitman, and S.D. Odintsov, Int. J. Mod. Phys. A 12 (1997) 4837; S.P. Gavrilov, D.M. Gitman, and A.E. Gonçalves, Int. J. Mod. Phys. A 16 (2001) 4235.

[41] N.B. Narozhny and A.I. Nikishov, Sov. J. Nucl. Phys. (USA) 11 (1970) 596.

[42] S.P. Gavrilov, J. Phys. A 39 (2006) 6407 arXiv:hep-th/0510093.

[43] S.A. Fulling, Phys. Rev. D 7 (1973) 2850; P.C.W. Davies, J. Phys. A 8 (1975) 609; W.G. Unruh, Phys. Rev. D 14 (1976) 870. 
[44] Y. Kluger, J.M. Eisenberg, and B. Svetitsky, Int. J. Mod. Phys. E 2 (1993) 333; F. Cooper, J.M. Eisenberg, Y. Kluger, E. Mottola, and B. Svetitsky, Phys. Rev. D 48 (1993) 190; J.C.R. Bloch et al, Phys. Rev. D 60 (1999) 116011; D.V.Vinnik et al, Eur. Phys. J. C 22 (2001) 341 arXiv:nucl-th/0103073.

[45] S.M. Schmidt et al., Int. J. Mod. Phys. E 7 (1998) 709; R.S. Bhalerao and G.C. Nayak, Phys. Rev. C 61 (2000) 054907.

[46] T. Lappi and L. McLerran, hep-ph/0602189; Nucl. Phys. A 772 (2006) 200.

[47] N.K. Nielsen and P. Olesen, Nucl. Phys. B 144 (1978) 376.

[48] J. Ambjorn and P. Olesen, Nucl. Phys. B 170 (1980) 60.

[49] C.A. Flory, Phys. Rev. D 28 (1983) 1425; Y.M. Cho, J.H. Kim, and D.G. Pak, Mod. Phys. Lett. A 21 (2006) 2789.

[50] B.L. Spokoiny, Sov. J. Nucl. Phys. 36 (1982) 277; Phys. Lett. A 88 (1982) 328 .

[51] A. Bialas, Phys. Lett. B 466 (1999) 301. 